\title{
The Origin of Gravity
}

\section{An Attempt to Answer this Question with the Help of Existing Concepts}

\author{
Hubert J. Veringa ${ }^{1}$ \\ ${ }^{1}$ Emeritus Professor at Eindhoven University, The Netherlands \\ Correspondence: Hubert J. Veringa, Emeritus Professor at Eindhoven University, The Netherlands. \\ E-mail: veringa48@planet.nl
}

Received: September 11, 2017

Accepted: October 8, 2017 Online Published: January 8, 2018

doi:10.5539/jmr.v10n1p67

URL: https://doi.org/10.5539/jmr.v10n1p67

\begin{abstract}
In this document an attempt is made to explain the origin of gravity. The basis for the analysis is a merger of quantum theory and relativity. Nowhere in the analysis there is any need to deviate from well proven and successful concepts of both theories and rules of calculation, and no exotic new particles will have to be introduced. By doing so it is demonstrated that, next to its local interactions of a multi-particle system, the Schrödinger equation leads to pairs of two and only two members. This solution is used as the invariant term in the quantized Einstein energy equation which finally leads to gravitational interactions between members of the pairs. With this particular solution for the quantum-mechanical wave function it is found that gravity is a second order effect operating over a long range. In this document it is tried to give a complete and consistent account of all steps that have been taken in the derivation of the classical Newton's gravity law. Further, the document emphasizes precise justification of some of the basic assumptions made and how it works out on a cosmological scale. It is also found that the generator of gravity is contributing mass to particles that have gravitational interaction.
\end{abstract}

Keywords: Gravity, Quantum Physics, Special Relativity, Dynamic mass, Cosmology

\section{Introduction}

In our daily life, gravity is experienced everywhere and at all moments. Without gravity the world as an entity would not exist, the Sun would not shine, water waves would not run, etc. Even if we would evaluate the consequences of a small change in the gravitational interaction, the universe would look different from how it is now. It is accepted as an inescapable force that keeps our existence together. However, where we have some basic understandings of the processes around us, there was not a suitable explanation for this force at a microscopic level.

Gravitational interaction manifests itself where other forces are not the determining factor. Therefore, in our real world, we see that our direct vicinity has structures of forms that are changing over short distances like mountains, cities, sky scrapers, boats, forests etc. At larger distances, of the order of 100 kilometers, the gravity becomes the dominant factor and bodies begin to take spherical shapes. Obviously, the smaller the gravity is, so to speak at smaller planets than earth, the structural variability will become larger. That the electromagnetic interaction becomes insignificant in shaping the environment is not due to the form of the electrostatic interaction, which has basically the same shape as the gravitational interaction, but it is due to the fact that positive and negative charges balance and compensate for their interaction. The influence of electromagnetism is becoming insignificant already at short distances.

Most of the theories on gravity start from gravity law as it is formulated centuries ago by Newton and see that its validity is beyond debate, at least at the scale which we can observe, but do not give insight into the basic mechanism behind this law. Now the general belief is that any suitable theory should include, or will be, a merger of classical quantum theory and relativity. Also the theory should allow for accommodation of the Higgs mechanism as a means to dedicate mass to any real particle. However, so far no theory that is widely accepted has been proposed. In the present document a new scheme of analysis for the mutual interaction between particles that have some exchange with respect to time and space will be presented. The remarkable thing is that, apparently for more than one reason, particles will be interacting in groups of two and only two and can give rise to gravitational interaction. This pair formation is described quantum mechanically. Either starting from the classical Schrödinger equation or the relativistic Einstein energy equation, but this latter formulated in a quantum mechanical setting known as the "Klein-Gordon" (KG) equation, results in the same wave function describing pairs of particles. Since this wave function represents a pair potential, a 
relativistic mass can be attributed to it which is used in the KG-equation to derive an interaction field between the members that form the ensemble. It is found that the right form of Newton's gravity law emerges by consequently working through the proposed schemes of both quantum mechanics and the basic equations of relativity theory as expressed by the quantum mechanical equivalent of the Einstein energy equation.

In the end of this paper a list of used symbols will be given for easy reference.

\section{The Special Relativity Theory}

At the beginning of the previous century it became apparent that the basic rules of mechanics shows some discrepancies when speeds are increasing. In daily life this was, however, not serious because the speeds at which discrepancies occur are far beyond the speeds which we are used to work with, but some remarkable facts, particularly when our understanding of cosmology increased, were observed which did not allow an explanation on the basis of classical Newtonian mechanics. Particularly when it became possible to measure with good accuracy the speed of light the peculiarities became even bigger. It was thought that the earth and light are moving through a stationary cosmological substance, the "ether frame", so that any measurement of the speed of light would depend on the direction at which we would measure it. It was the Michelson-Morley experiment, (Ney, 1965) that showed that, whatever we try, we will always find the same value of the speed of light: $c=3 \times 10^{8} \mathrm{~m} / \mathrm{sec}$.

This unexpected result remained puzzling for some years but later it was realized that also the laws of electromagnetic are entirely independent of the speed and place of the observer.

So if we have a frame of reference, a coordinate system, in which the observer is situated, $\Sigma$, and a moving one travelling with speed $v$, which we call $\Sigma^{\prime}$, the distance between points in either system remains the same. As the information about this distance is based on visual observation of things which are happening, we can conclude that if the coordinates, including time, in $\Sigma$ are $\mathrm{x}, \mathrm{y}, \mathrm{z}$ and $\mathrm{t}$ and in $\Sigma^{\prime}$ they are $x^{\prime}, y^{\prime}, z^{\prime}$ and $t^{\prime}$, the following relation must hold:

$$
x^{2}+y^{2}+z^{2}-c^{2} t^{2}=x^{\prime 2}+y^{\prime 2}+z^{\prime 2}-c^{2} t^{\prime 2} .
$$

Lorentz proposed on the basis of this invariance a scheme of transformations between the coordinates in $\Sigma$ and $\Sigma$ ' to guarantee that this invariance is always valid. These transformations, known as the Lorentz transformations, can be derived from this equation (2.1) and read:

$$
x=\gamma\left(x^{\prime}+v t^{\prime}\right), y=y^{\prime}, z=z^{\prime}, c t=\gamma\left(c t^{\prime}+v x^{\prime} / c\right),
$$

with: $\gamma=1 / \sqrt{1-v^{2} / c^{2}}$. Einstein realized that there is more in this than just a few transformation rules and generalized this idea by proposing the concept of "four-vectors". Four-vectors are mathematical objects in four dimensional space which transform according to the Lorentz rules and therefore have the Lorentz invariance as in equation (2.1). Such a four-vector is symbolized as $\{x, y, z, c t\}$.

If we now have two four-vectors: $\left\{a_{1}, b_{1}, c_{1}, d_{1}\right\}$ and $\left\{a_{2}, b_{2}, c_{2}, d_{2}\right\}$, the invariants are:

$$
a_{1}^{2}+b_{1}^{2}+c_{1}^{2}-d_{1}^{2}=C_{1} \text { and: } a_{2}^{2}+b_{2}^{2}+c_{2}^{2}-d_{2}^{2}=C_{2},
$$

and therefore:

$$
\left(a_{1}^{2}+a_{2}^{2}\right)+\left(b_{1}^{2}+b_{2}^{2}\right)+\left(c_{1}^{2}+c_{2}^{2}\right)-\left(d_{1}^{2}+d_{2}^{2}\right)=C_{1}+C_{2} .
$$

But also the vectorial product of two four-vectors is invariant so that:

$$
a_{1} a_{2}+b_{1} b_{2}+c_{1} c_{2}-d_{1} d_{2}=C_{3},
$$

and therefore the sum of two four-vectors again is a four-vector $\left\{a_{1}+a_{2}, b_{1}+b_{2}, c_{1}+c_{2}, d_{1}+d_{2}\right\}$ with the invariant:

$$
\left(a_{1}+a_{2}\right)^{2}+\left(b_{1}+b_{2}\right)^{2}+\left(c_{1}+c_{2}\right)^{2}-\left(d_{1}+d_{2}\right)^{2}=C_{1}+C_{2}+C_{3} .
$$

The multiplication rule (2.3d) makes equations (2.3c) and (2.3e) equivalent which will be used in the microscopic theory (paragraph 7) and for the translation to the gravitationally interacting macroscopic bodies (paragraph 8).

There are many different four-vectors, particularly in electrodynamics, but for the present theory we will only need, next to the one mentioned, the momentum-energy vector $\left\{p_{x}, p_{y}, p_{z}, E / c\right\}$ with the invariance:

$$
p_{x}^{2}+p_{y}^{2}+p_{z}^{2}-E^{2} / c^{2}=C .
$$

With the help of the famous mass-energy equation of Einstein: $E=\gamma m_{0} c^{2}$ this invariance is readily rewritten as an equation that will be of great importance for the rest of this document:

$$
E^{2}-p^{2} c^{2}=m_{0}^{2} c^{4} .
$$


When we substitute $E=T_{k}+m_{0} c^{2}$ into equation (2.5) and take the limit of $c$ to infinity we get the more familiar relation $T_{k}=p^{2} / 2 m$ with $m$ for the mass instead of $m_{0}$. Clearly we have to incorporate any other form of energy, like the potential energy, into $m_{0}$.

The gradient operator $\left\{\frac{\partial}{\partial x}, \frac{\partial}{\partial y}, \frac{\partial}{\partial z}, \frac{1}{c} \frac{\partial}{\partial t}\right\}$ is a special one with the invariance, but applied to a field $\varphi$ looks like:

$$
\left(\frac{\partial^{2}}{\partial x^{2}}+\frac{\partial^{2}}{\partial y^{2}}+\frac{\partial^{2}}{\partial z^{2}}-\frac{1}{c^{2}} \frac{\partial^{2}}{\partial t^{2}}\right) \varphi=C \varphi
$$

If $\varphi$ is a scalar field it is just the multiplication of the invariant of the gradient operator with the value $\varphi$. If $\varphi$ is a vector field the equation applies to the separate components of this vector field. This equation (2.6) is the well known equation describing the movement of a wave through a medium. If this medium is the vacuum, we can set $C=0$, and signals move at the speed of light as the general solution is:

$$
\varphi=f(x-c t) g(y-c t) h(z-c t) .
$$

In which $f, g$ and $h$ are arbitrary functions in the arguments as indicated.

Due to the fact that equation (2.6) is derived from the invariant, we can replace the space coordinates $x, y, z$ and time $t$ in $\Sigma$ by the Lorentz transformed ones: $x^{\prime}, y^{\prime}, z^{\prime}$ and $t^{\prime}$ in $\Sigma^{\prime}$ and therefore also in the solution (2.7). The consequence is then that observers in $\Sigma$ and $\Sigma^{\prime}$, watching the same event, will conclude that there is the same value of $c$. This may look as a surprising conclusion but it has already been assumed implicitly in the Lorentz transformation rules.

The famous Lectures on Physics by Feynman (Feynman et. al,, 1965) give a very good introduction into four-vectors as they are dealt with in this paragraph. Further reading on Relativity is recommended in Ney's short study on electromagnetism and Relativity (Ney, 1965).

\section{Quantum Rules}

In quantum theory the behavior of a microscopic particle in space and time is described by a wave function, denoted by $\psi$. This wave function is a mathematical expression in space and time, usually in complex notation. The product of this wave function and its complex conjugated $\psi^{*}: \psi \psi^{*}$ is the probability that a particle can be found at that particular place and moment.

Quantum mechanics relies largely on operators. Operators are mathematical abstractions that do something with a wave function. In the first place a wave function is a solution of the operator equation like, as an example for the momentum:

$$
\widehat{p_{x}} \psi=\frac{\hbar}{i} \frac{\partial}{\partial x} \psi=p \psi
$$

The expectation value of the momentum in this example then is given by:

$$
P=\int \psi^{*} \widehat{p_{x}} \psi d V
$$

with the integration over the entire space where the operator is active. As a consequence of this the normalization of a wave function is:

$$
\int \psi^{*} \psi d V=1
$$

The famous Schrödinger equation derives from the basic rule based on the Hamiltonian and energy operator acting on this wave function and gives the energy as a function of momentum and space as a solution. In short it reads:

$$
\hat{\mathrm{E}}(t) \psi(x, y, z, t)=\hat{\mathrm{H}}\left(\widehat{p_{x}}, \widehat{p_{y}}, \widehat{p_{z}}, x, y, z\right) \psi(x, y, z, t) .
$$

In this equation the time dependence is allocated to the energy operator by $\hat{\mathrm{E}}(t)=i \hbar \partial / \partial \mathrm{t}$, while the dependences on momentum and place are allocated to the Hamiltonian. The momentum in the Hamiltonian is, obviously, also an operator and given by $\widehat{p_{x}}=-i \hbar \partial / \partial \mathrm{x}$, and similarly for the $y$ - and $z$-coordinates. It is worthwhile to note that the conjugated wave function is a solution of the conjugated Schrödinger equation and not the result of an operation on the wave function itself.

For the development of the present model we can write the Hamiltonian more explicitly as:

$H=\left(p_{x}^{2}+p_{y}^{2}+p_{z}^{2}\right) / 2 m+V(x, y, z)$ or as operators:

$$
\hat{\mathrm{H}}\left(\widehat{p_{x}}, \widehat{p_{y}}, \widehat{p_{z}}, x, y \cdot z\right)=-\frac{\hbar^{2}}{2 m}\left(\frac{\partial^{2}}{\partial x^{2}}+\frac{\partial^{2}}{\partial y^{2}}+\frac{\partial^{2}}{\partial z^{2}}\right)+V(x, y, z) .
$$


This equation has been very successful in describing the behavior of microscopic particles in their local environment. Such a local environment can be anywhere: in open space as well as in structures like a solid. But once speeds are getting higher, it is found that the validity of this equation can break down.

For this reason another energy equation which better fulfills the relativistic behavior is proposed based on the equation (2.4), but now in the form of operators:

$$
-\hbar^{2}\left(\frac{\partial^{2}}{\partial t^{2}}-c^{2}\left(\frac{\partial^{2}}{\partial x^{2}}+\frac{\partial^{2}}{\partial y^{2}}+\frac{\partial^{2}}{\partial z^{2}}\right)\right) \varphi(x, y, z, t)=m_{0}^{2} c^{4} \varphi(x, y, z, t) .
$$

This equation is the relativistic alternative for the Schrödinger equation. It is called the "Gordon-Klein" equation. From the form it can be seen that the wave function has the character of a travelling wave in open space. If there is no mass, mo $=0$, it will propagate with the speed of light $c$. With mass the propagation speed will always be lower than the speed of light.

Both wave equations (3.4) and (3.6) with the Hamilton operator in (3.5) will be used in the further development of the theory. If we apply the function:

$$
\varphi(x, y, z, t)=\psi(x, y, z, t) e^{i m_{0} c^{2} t / \hbar}=\psi(x, y, z) e^{i\left(E+m_{0} c^{2}\right) t / \hbar}
$$

as a solution of equation (3.6) we get back the Schrödinger equation (3.5) in the limit of $c$ to infinity, similarly as in the previous paragraph. In the present context it means that a solution of the KG-equation for $c$ to infinity becomes a solution of the Schrödinger equation. It does not necessarily mean that the Schrödinger equation has limited validity. We will come back to this point in the end of paragraph 5 .

Most surprising, is that, even in cases where relativistic effects should manifest, the Schrödinger equation and non relativistic quantum mechanical interpretations can keep their validity. The Schrödinger equation derives in the limit of $c$ to infinite and, therefore, all interactions due to this Schrödinger equation occur instantaneously which is not possible according to the relativity theory. This is the well known difficulty of the reconciliation of relativity theory and quantum mechanics for which, so far, no solution has been found. In paragraph 11 a clear example of this phenomenon, which is also relevant for the gravity theory, will be discussed.

For the development of the theory of gravity one important remark has to be made about the use of operators. The expectation value, or the probability that this mass shows up at the location $x, y, z$ and at time $t$ due to the wave function $\psi(x, y, z, t)$ is found by considering the mass as an operator:

$$
m(x, y, z, t)=\psi^{*}(x, y, z, t) m(x, y, z, t) \psi(x, y, z, t) .
$$

Although this looks self-evident, it however is not. The equation (3.6) concerns the square of the rest mass distribution $m_{0}^{2}(x, y, z . t)$ and the expectation value is:

$$
m_{0}^{2}(x . y, z, t)=\psi^{*}(x, y, z, t) m_{0}^{2}(x, y, z, t) \psi(x, y, z, t) .
$$

To finish these more general rules in this paragraph one more thing has to be done. As we are dealing with particles which do not have a geometrical structure we can adopt spherical symmetry throughout the development of the theory. For this reason the coordinates $\mathrm{x}, \mathrm{y}$, and $\mathrm{z}$ are not so practical. It would be more easy to work with coordinates which have the same symmetry as the particles and their environment. The main issue therefore is to cast the operator ${\widehat{p_{x}}}^{2}+{\widehat{p_{y}}}^{2}+{\widehat{p_{z}}}^{2}$ into a more appropriate form involving spherical symmetry so that the only coordinate $r$ is necessary.

This is first done for a system of one particle, starting from the $x-, y-, z$-component of the gradient operator $(g r a d)_{x} \psi$, $(\operatorname{grad})_{y} \psi,(\operatorname{grad})_{z} \psi$, only taking $r$-dependences:

$$
(\operatorname{grad})_{x} \psi=\frac{\partial \psi}{\partial x}=\frac{\partial \psi}{\partial r} \frac{\partial r}{\partial x}=\frac{x}{r} \frac{\partial \psi}{\partial r}
$$

and differentiating again:

$$
(\operatorname{grad})_{x}(\operatorname{grad})_{x} \psi=\frac{\partial^{2} \psi}{\partial x^{2}}=\frac{1}{r} \frac{\partial \psi}{\partial r}-\frac{x^{2}}{r^{3}} \frac{\partial \psi}{\partial r}+\frac{x^{2}}{r^{2}} \frac{\partial^{2} \psi}{\partial r^{2}}
$$

and the same for the $y$ - and $z$ - coordinates. We find, remembering that $x^{2}+y^{2}+z^{2}=r^{2}$ :

$$
\left(\frac{\partial^{2}}{\partial x^{2}}+\frac{\partial^{2}}{\partial y^{2}}+\frac{\partial^{2}}{\partial z^{2}}\right) \psi=\left(\frac{\partial^{2}}{\partial r^{2}}+\frac{2}{r} \frac{\partial}{\partial r}\right) \psi=\frac{1}{r^{2}} \frac{\partial}{\partial r} r^{2} \frac{\partial \psi}{\partial r}
$$

But for a system of two particles, labelled $i$ and $j$ we have to use vector notation for the gradients working on the wave 
function. This wave function describes in one expression the set of the $i$ and $j$ particle. We first take the gradient for particle $i$ as before:

$$
(\operatorname{grad})_{x_{i}} \psi=\frac{\partial \psi}{\partial x_{i}}=\frac{\partial \psi}{\partial r_{i}} \frac{\partial r_{i .}}{\partial x_{i}}=\frac{x_{i}}{r_{i}} \frac{\partial \psi}{\partial r_{i}},
$$

and the same for the $\mathrm{y}$ - and $\mathrm{z}$ - component of the coordinates of particle $i$ and $j$. So we have in the $r_{i}$ - direction:

$$
(\operatorname{grad})_{r_{i}} \psi=\frac{\partial \psi}{\partial r_{i}}
$$

Applying the same procedure for gradient in the $r_{j}$ - direction we get the final result:

$$
(\operatorname{grad})_{r_{i}} \psi(\operatorname{grad})_{r_{j}} \psi=\frac{\partial \psi}{\partial r_{i}} \frac{\partial \psi}{\partial r_{j}} .
$$

Although these rules are not particularly quantum rules, they will be important for the development of the quantum-based theory.

For the further development of the theory, however, one other aspect has to be paid attention to. That is how a particle, which is part of a specific structure like a solid, can be treated. The operators mentioned until now have particular emphasis for freely moving particles, but it is obvious that also particles constituting larger entitles do show gravitational interaction.

From the basic concept of quantum mechanics we know that particles do have a non zero probability to show up anywhere in space. This probability can be very small and for the determination of its behavior in its local environment it is so small that it is usually neglected. But for gravitational interaction, which in essence is extremely small compared to any other force, it is of relevance.

To investigate this problem we therefore construct a wave function that combines its local behavior with its global one. The total wave function describing a particle under its local influences, $\psi_{l o c}\left(r_{l o c}, t\right)$, and its extension in free space, $\psi_{\text {inf }}\left(r_{\text {inf }}, t\right)$, is given by: $\psi_{\text {tot }}=\psi_{\text {loc }} \psi_{\text {inf }}$. The coordinate $r_{l o c}$ is the position of the centre-of-mass of the particle inside the atom or nucleus or a solid object and the coordinate, $r_{\text {inf }}$, is the position of the particle from the point of view of an outside observer. They therefore are mutually independent. In the same way we define, as before, the Hamilton operator as: $\widehat{H}_{\text {tot }}=\left\{\hat{p}^{2}\right\}_{l o c} / 2 m_{l o c}+\left\{\hat{p}^{2}\right\}_{\text {inf }} / 2 m_{\text {inf }}+V_{l o c}\left(r_{l o c}\right)+V_{\text {inf }}\left(r_{\text {inf }}\right)$. The masses $m_{l o c}$ and $m_{\text {inf }}$ are not necessarily the same. The $m_{\text {inf }}$ is the mass to be connected to the particle as it can move freely around whereas $m_{l o c}$ is the mass of the particle under the influence of the local interactions, sometimes called "reduced mass". It follows that:

$$
\begin{aligned}
\widehat{H}_{\text {tot }} \psi_{\text {tot }} & =\left(\left\{\hat{p}^{2}\right\}_{l o c} / 2 m_{l o c}+\left\{\hat{p}^{2}\right\}_{\text {inf }} / 2 m_{\text {inf }}+V_{\text {loc }}+V_{\text {inf }}\right)\left(\psi_{\text {loc }} \psi_{\text {inf }}\right)= \\
& =\left(\left\{\hat{p}^{2}\right\}_{l o c} / 2 m_{l o c}+V_{l o c}\right) \psi_{\text {loc }} \psi_{\text {inf }}+\left(\left\{\hat{p}^{2}\right\}_{\text {inf }} / 2 m_{\text {inf }}+V_{\text {inf }}\right) \psi_{\text {loc }} \psi_{\text {inf }} .
\end{aligned}
$$

Separating the local effect from the surroundings we can set:

$$
\begin{aligned}
& \left(\left\{\hat{p}^{2}\right\}_{l o c} / 2 m_{l o c}+V_{l o c}\right) \psi_{l o c}=E_{l o c} \psi_{l o c} \text { and: } \\
& \left(\left\{\hat{p}^{2}\right\}_{\text {inf }} / 2 m_{\text {inf }}+V_{\text {inf }}\right) \psi_{\text {inf }}=E_{\text {inf }} \psi_{\text {inf }} .
\end{aligned}
$$

The first equation (3.12a) is the Schrödinger equation describing the behavior of the particle in its local environment like in the nucleus or a solid where it has its individual interactions. The second equation (3.12b) describes its movement or presence in the outer space in which the particle, or as part of a larger entity, can move around. By taking $V_{\text {inf }}$ as a constant it is assumed that the behaviour out of its local influences is taken into consideration. This second equation is the starting point in the development of the theory in the next paragraphs. The splitting up as in equation (3.12a) and (3.12b) disconnects the local interaction of separate particles, as is normally done in quantum mechanics, from the movement or presence of the particle individually or as part of a larger entity. In what follows we will only consider the second equation as this gives the generator for the gravitational interaction. As we are interested in the effects of masses outside the local interactions we will from now on take for the mass $m_{\text {inf }}$ the quantity $m$, as it will also be the case for the coordinate.

More on this subject can be read in Ney (Ney, 1965), Messiah (Messiah, 1985) and Heitler (Heitler, 1945). 


\section{Groups of Particles and Sub-spaces}

Gravity is an attractive force between two bodies, or, at a microscopic level, two particles and therefore any theory will have to account for multi-particle systems. The previous paragraph has shown that the Hamiltonian will be the central operator but in that representation it only accounts for single entities. For the development of the theory we have to modify the Hamiltonian for multi-particle systems.

The most simple expression for the kinetic energy in the Hamiltonian for a group of particles numbered by $k$ is given by:

$$
\hat{p}^{2} / 2 m=\sum_{k}{\widehat{p_{k}}}^{2} / 2 m_{k} .
$$

This expression does, however, not clearly enough describe the behavior of particle interaction as members of a group, but it will be shown that an alternative representation is possible in which still the total kinetic energy remains the same. The starting point is to write equation slightly differently

$$
\frac{\hat{p}^{2}}{2 m}=\sum_{k}{\widehat{p_{k}}}^{2} / 2 m_{k}=\sum_{k}\left(\widehat{p_{k}} / \sqrt{2 m_{k}}\right)^{2} .
$$

This equation does not look so special, but it shows that, if we want to modify the kinetic energy in the Hamiltonian, we will have to perform our analysis in the $\widehat{p_{k}} / \sqrt{2 m_{k}}$-space. For reasons that will become clear later we will now modify the Hamiltonian for the two-particle ensemble $(i j)$ and refer to Figure 1.

In Figure 1 particles $m_{i}$ and $m_{j}$ are moving with momenta $p_{i}$ and $p_{j}$. But we are interested in their behaviour in the space as seen from point $\mathrm{O}_{2}$ and therefore we apply the cosine-rule to both triangles [1] and [2]. Knowing that:

$\cos \delta_{2}=-\cos \left(180-\delta_{2}\right)$ and taking $p_{i j} / \sqrt{2 m_{i}}=p_{j i} / \sqrt{2 m_{j}}$, it follows that:

$$
\widehat{p}_{l}^{2} / 2 m_{i}+{\widehat{p_{j}}}^{2} / 2 m_{j}={\widehat{p_{g}}}^{2} / 2 m_{g}+{\widehat{p_{l \jmath}}}^{2} / 2 m_{i}+{\widehat{p_{\jmath l}}}^{2} / 2 m_{j} \text {. }
$$

In this modified kinetic energy part of the Hamiltonian the first term at the right hand is the kinetic energy of the group, identified with label $g$, consisting of $m_{i}$ and $m_{j}$ with mass $m_{g}=m_{i}+m_{j}$ and moving as one single entity. The second term is the kinetic energy in the sub-space. The group momentum vector $p_{g} / \sqrt{2 m_{g}}$ is not equal to any of the other ones so that $\widehat{p_{g}}$ has to be defined separately. As the interaction between the two particles is only within the sub-space $r_{i j}$, we will not have to bother about this first term at the right hand. This is fortunate because it depends on the angle between $\widehat{p}_{l} / \sqrt{2 m_{i}}$ and $\widehat{p}_{j} / \sqrt{2 m_{j}}$ which would severely complicate the problem. It can also be seen that this modification of the Hamiltonian only works well for two particles as the geometrical argument is confined to one plane. More particles would compel us to perform the analysis in many more different planes and would not give a tractable solution.

Another important observation is that because $p_{i j} / \sqrt{2 m_{i}}=p_{j i} / \sqrt{2 m_{j}}$ the sub-space is symmetric from the point of view of an observer in $\mathrm{O}_{2}$. This issue of symmetry will come back in the solution of the Schrödinger equation with the modified Hamiltonian.

We will now extend the modified Hamiltonian equation, obtained by substituting equation (4.3) into (4.2), for more than two particles, but all of them interacting in groups of two and only two:

$$
\sum_{k}{\widehat{p_{k}}}^{2} / 2 m_{k}=1 / N\left(\sum_{g}{\widehat{p_{g}}}^{2} / 2 m_{g}+1 / 2 \sum_{i \neq j}\left({\widehat{p_{l j}}}^{2} / 2 m_{i}+{\widehat{p_{l l}}}^{2} / 2 m_{j}\right)\right) \text {. }
$$

The $N$-factor, the number of particles, is necessary as in the summation each particle is counted $N$ times. The pairs are counted by the $g$-index. Later, when the analysis brings us to the final result, we will come back to the group momentum and evaluate the consequence of its dependence on the momenta of $m_{i}$ and $m_{j}$.

For completeness we will now derive this dependence but come back to it later. For this we apply the cosine-rules from the corner $\mathrm{O}_{1}$ for the triangles [1] and [2] separately and together: [1+2]. the equations are:

$$
\begin{gathered}
\left(\widehat{p_{l j}} / \sqrt{2 m_{i}}+\widehat{p_{\jmath l}} / \sqrt{2 m_{j}}\right)^{2}={\widehat{p_{l}}}^{2} / 2 m_{i}+{\widehat{p_{J}}}^{2} / 2 m_{j}-2 \widehat{p_{l} \widehat{p}_{j}} \cos \delta_{1} / \sqrt{4 m_{i} m_{j}}, \\
{\widehat{p_{g}}}^{2} / 2 m_{g}={\widehat{p_{l}}}^{2} / 2 m_{i}+{\widehat{p_{J}}}^{2} / 2 m_{j}-{\widehat{p_{l j}}}^{2} / 2 m_{i}-{\widehat{p_{\jmath l}}}^{2} / 2 m_{j} .
\end{gathered}
$$




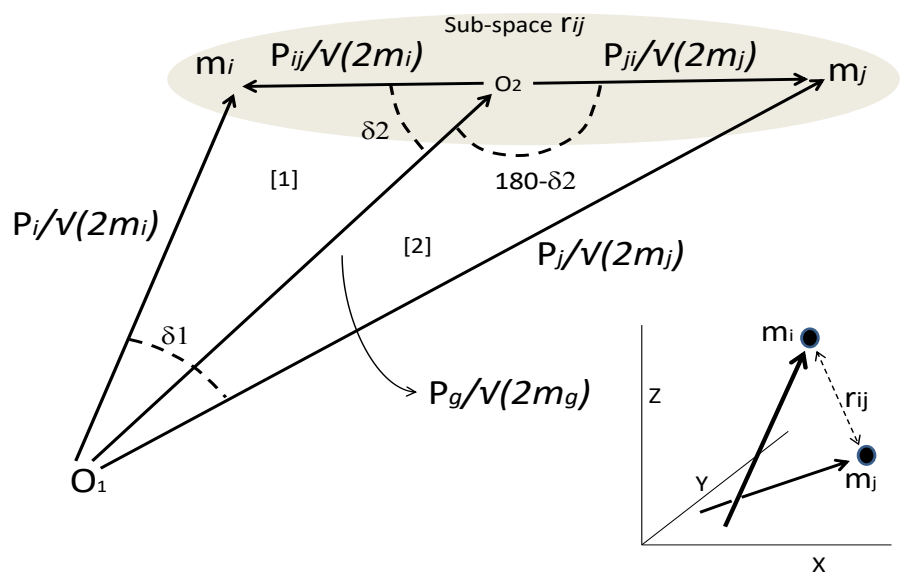

Figure 1. The relation (4.3) found by applying the cosine-rule to both triangles [1] and [2] if the lengths of the arrows $p_{i j} / \sqrt{2 m_{i}}$ and $p_{j i} / \sqrt{2 m_{j}}$ are the same. In this view vectors and operators are treated as equivalent. Note that in the two-dimensional momentum space the velocity or the momentum vectors for particles always have the same origin.

This cosine factor showing up complicates the analysis, but in the end it will not trouble our analysis as it can be circumvented.

Now also the analysis will have to be repeated starting from the equation (2.4), but as it is only dealing with the momenta, the result of the previous analysis can be used if we simply replace the vector $p_{k} / \sqrt{2 m_{k}}$ by $c m_{k} \sqrt{\gamma_{k}^{2}-1}$ with the $k$-label representing $i, j, g$ and $p_{i j}, p_{j i}$ unchanged. The analysis will be continued in paragraph 11 .

\section{The Sub-space in More Detail}

Starting from the unmodified Hamiltonian, the general solution of a wave equation describing independent particles in spherical symmetry is initiated by the operator $\hat{p}^{2} / 2 m=\sum_{k} \hat{p}_{k}^{2} / 2 m_{k}$, and reads:

$$
\psi=. . \psi_{i} \psi_{j} \ldots \psi_{l}=. .\left(\frac{\alpha_{i}}{r_{i}}\right) e^{i \beta_{i} r_{i}} x\left(\frac{\alpha_{j}}{r_{j}}\right) e^{i \beta_{j} r_{j}} x \ldots x\left(\frac{\alpha_{l}}{r_{l}}\right) e^{i \beta_{l} r_{l}} x \ldots=\prod_{k}\left(\frac{\alpha_{k}}{r_{k}}\right) e^{i \beta_{k} r_{k}} .
$$

For this equation the equations (3.4), (3.5), and (3.10) have been used. But we have regrouped the kinetic contribution to the Hamiltonian for the same set of particles as:

$$
\frac{\hat{p}^{2}}{2 m}=\sum_{k} \hat{p}_{k}^{2} / 2 m_{k}=1 / N\left(\sum_{g} \hat{p}_{g}^{2} / 2 m_{g}+1 / 2 \sum_{i \neq j}\left(\hat{p}_{i j}^{2} / 2 m_{i}+\hat{p}_{j i}^{2} / 2 m_{j}\right)\right),
$$

and first we will only consider the second part of it at the right hand side, to start with the group (ij) of two particles only, thus we restrict ourselves to the sub-space with coordinates $r_{i j}$.

Per group there are two independent particles. For the group under consideration like in Figure 2, it is indicated by the masses $m_{i}$ and $m_{j}$. and they experience some force reflected by the potential $V_{i}$ and $V_{j}$. Spherical symmetry is next adopted and the only boundary condition is that the wave function is zero at infinity. 


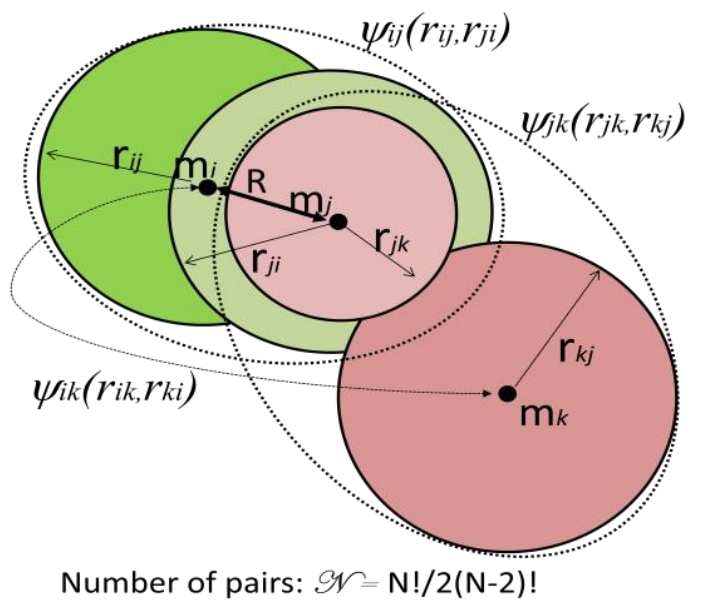

Figure 2. Forming and describing of $\mathcal{N}=N ! / 2(N-2)$ ! Pairs. In this example the number of groups is three.

We have per pair one coordinate system $r_{i j}$ around $m_{i}$ and one $r_{j i}$ around $m_{j}$. In this way an observer at a distance $r_{i j}$ from particle $m_{i}$ and at $r_{j i}$ from particle $m_{j}$ will see that the total wave equation of the individual pair $(i j)$ is defined as follows (Messiah, 1985):

$$
\widehat{H_{l j}} \psi_{i j, t}=i \hbar \frac{\partial}{\partial t} \psi_{i j, t}=-\left(\frac{\hbar^{2}}{2 m_{i}} \frac{1}{r_{i j}^{2}} \frac{\partial}{\partial r_{i j}} r_{i j}^{2} \frac{\partial}{\partial r_{i j}}+\frac{\hbar^{2}}{2 m_{j}} \frac{1}{r_{j i}^{2}} \frac{\partial}{\partial r_{j i}} r_{j i}^{2} \frac{\partial}{\partial r_{j i}}\right) \psi_{i j, t}+\left(V_{i}+V_{j}\right) \psi_{i j, t} .
$$

$\psi_{i j, t}$ is the time and space dependent wave function. The time dependence can be removed by replacing the time dependent wave function $\psi_{i j, t}$ by $\psi_{i j} e^{i E_{i j} t / \hbar}$. Further, define $V_{i}+V_{j}$ by $V_{i j}$ and we get:

$$
\left(E_{i j}-V_{i j}\right) \psi_{i j}+\frac{\hbar^{2}}{2 m_{i}} \frac{1}{r_{i j}^{2}} \frac{\partial}{\partial r_{i j}} r_{i j}^{2} \frac{\partial}{\partial r_{i j}} \psi_{i j}+\frac{\hbar^{2}}{2 m_{j}} \frac{1}{r_{j i}^{2}} \frac{\partial}{\partial r_{j i}} r_{j i}^{2} \frac{\partial}{\partial r_{j i}} \psi_{i j}=0 .
$$

To simplify the equation replace $E_{i j}-V_{i j}$ by $\varepsilon_{i j}$ to propose a solution that is valid in areas where the $V_{i j}$ is not of great influence anymore as follows:

$$
\psi_{i j}=\left(\frac{\alpha_{i j}}{r_{i j}}+\frac{\alpha_{j i}}{r_{j i}}\right) e^{i \beta_{i j} r_{i j}+i \beta_{j i} r_{j i}},
$$

where $\alpha_{i j}$ and $\beta_{i j}$ are constants independent of space coordinates and time. This solution means that we consider the wave function outside the surroundings where the potential energy with all its peculiarities has a very minor effect on the shape of the wave function. The only interaction that can play a role will then be based solely on gravitational interaction. By substituting the solution in equation (5.3) the following relation is found:

$$
\begin{aligned}
- & \frac{\hbar^{2} i}{r_{i j} r_{j i}}\left(\frac{\alpha_{i j} \beta_{j i}}{m_{j}}+\frac{\alpha_{j i} \beta_{i j}}{m_{i}}\right) e^{i \beta_{i j} r_{i j}+i \beta_{j i} r_{j i}}-\frac{\hbar^{2}}{2}\left(\frac{\beta_{i j}^{2}}{m_{i}}+\frac{\beta_{j i}^{2}}{m_{j}}\right) x \\
& x\left(\frac{\alpha_{i j}}{r_{i j}}+\frac{\alpha_{j i}}{r_{j i}}\right) e^{i \beta_{i j} r_{i j}+i \beta_{j i} r_{j i}}+\varepsilon_{i j}\left(\frac{\alpha_{i j}}{r_{i j}}+\frac{\alpha_{j i}}{r_{j i}}\right) e^{i \beta_{i j} r_{i j}+i \beta_{j i} r_{j i}}=0 .
\end{aligned}
$$

The complex first term at the left hand side is to be set to zero and in a pair-wise process $\alpha_{i j} \beta_{j i} / m_{j}+\alpha_{j i} \beta_{i j} / m_{i}=0$ and $\beta_{i j}^{2} \hbar^{2} / 2 m_{i}+\beta_{j i}^{2} \hbar^{2} / 2 m_{j}=\varepsilon_{i j}=\sigma_{0}\left(m_{i}+m_{j}\right)$ so that for every value of the energy there will be a value for $\sigma_{0}$ and the $\beta$ 's can adapt themselves. Therefore, whatever is the situation in which $\mathrm{m}_{\mathrm{i}}$ and $\mathrm{m}_{\mathrm{j}}$ find themselves, there is always a $\beta_{j i}$ and a $\beta_{i j}$ and they have no influence on the $\alpha^{\prime} s$ as long as $\alpha_{i j}=\alpha_{j i}$. It means, that the interaction occurs in the sub-space with a pair to be considered as one single entity with a mass of $\left(m_{i}+m_{j}\right)$ and, apart from the separation between the members of the pair (R), independent of the situation these members are in. Further, it has to be noticed that the Schrödinger equation based on the modified Hamiltonian only is possible for groups of two and only two particles. This conclusion has already been drawn in a slightly different way in the previous paragraph where the geometrical argument in momentum space is only possible for two partices with momenta vectors in one plane.

We already came across the fact that the sub-space $r_{i j}$ in momentum space for the observer in $\mathrm{O}_{2}$ in Figure 1 is symmetric and therefore the solution $\Psi\left(\alpha_{i j}, \alpha_{j i}\right)$ is symmetric, meaning, again, that $\alpha_{i j}=\alpha_{j i}$. 
At the moment not much is known about the $\alpha^{\prime} s$, but one requirement to be imposed on the wave function is that it represents a pair of particles. For the time being it can be said that:

$i$. The $\alpha^{\prime} s$ cannot depend on the running variables in the wave equation: $r_{i j}$ or $t$. It will be a constant that can only depend on fundamental nature constants and the particle masses.

ii. It should make no difference for the outside world how one member sees its partner or whether and how we see the two members of the pair. It means that we can say: $\alpha_{i j}=f\left(m_{i}\right) f\left(m_{j}\right)$.

iii. There is no pair if either $m_{i}$ or $m_{j}$ equals zero so that $f\left(m_{i}\right)=0$ for $m_{i}=0$ and the pair potential should increase linearly with both participating masses in the pair.

To sum up also the movement of the group as one entity and the fact that there are $N$ particles and $\mathcal{N}=N ! / 2(N-2)$ ! Pairs leads to a total wave function as:

$$
\psi=\prod_{i j}\left(\frac{\alpha_{i j}}{r_{i j}}+\frac{\alpha_{j i}}{r_{j i}}\right) e^{i \beta_{i j} r_{i j}+i \beta_{j i} r_{j i}} \prod_{g}\left(\frac{\alpha_{g}}{r_{g}}\right) e^{i \beta_{g} r_{g}}
$$

The second product is due to the first contribution to the momentum-based energy term in equation (4.4) and, as already mentioned, it generates no gravitational interaction. The index $g$ is identified by the pair ( $i j$ ) as indicated in figure 1 . The first one in the product (5.6) gives gravitational interaction in the case of two, and only two members in an ensemble where the sum is taken over all possible and unique pairs $(i j)$. As the pairs exist in their own unique coordinate system $r_{i j}, r_{j i}$ there is no reason to consider all the pairs together but only the behavior of a single pair. In the end we will add up all the contributions of the pairs as shown schematically in Figure 5 in paragraph 8.

There is freedom in the choice of the particles $m_{i}, m_{j},---, m_{l},---$. It can actuallly be anything like elementary particles, nuclei or even larger entities if, at least, we can describe such an entity by a single wave function in its own coordinate system and solve the equation to form a pair with another entity.

Later it will be confirmed that, as before and for the sake of symmetry in the mutual gravitational interaction, the two $\alpha^{\prime} s$ should be equal. It also means that the $\beta$ 's have opposite signs and fixed values and, by taking the $\alpha^{\prime} s$ equal, we make their values independent of the masses and the energies of the members of the pair. The $\varepsilon_{i j}$ could have been split into two separate quantities as $\varepsilon_{i j}$ and $\varepsilon_{j i}$ to dedicate the $\beta_{i j}^{2}$ and $\beta_{j i}^{2}$-values to the separate energies of the two particles.

It is also interesting to notice that the solution of the wave equation for the pairs like in equation (5.6) looks different from a solution for a single particle on the basis of the unmodified Hamiltonian as in equation (5.1). For instance, if we take a look at the $r_{i j}$ dependence in the solution (5.6), we see that there is an extra $r_{i j}$ dependent factor in the exponential term. This latter term is insufficient to make such a solution applicable for the operator working on $r_{i j}$. For it to be sufficient we need the total pre-exponential factor as given in equation (5.6).

The second approach is taking the KG-equation as the starting point. In this way we guarantee full co-variance throughout the entire analysis. The equation has been given already and reads: $E^{2}-p^{2} c^{2}=m_{0}^{2} c^{4}$ or expressed alternatively: $E^{2} / m_{0}^{2} c^{4}-p^{2} / m_{0}^{2} c^{2}=1$, and translated into quantum mechanical language for an ensemble of two particles:

$$
\left.\left(E_{i j}^{2} / m_{0 i}^{2} c^{4}-E_{j i}^{2} / m_{0 j}^{2} c^{4}\right) \varphi_{i j}-\left(\left(\widehat{p_{l j}}\right)^{2} / m_{0 i}^{2} c^{2}-\left(\widehat{p_{j l}}\right)^{2} / m_{0 j}^{2} c^{2}\right)\right) \varphi_{i j}=0,
$$

where ${\widehat{p_{l j}}}^{2}$ is the square of the momentum operator in spherical coordinates as in equation (4.3) and $m_{0 i}, m_{0 j}$ the rest mass of the particle $i, j$ in the ensemble $(i j)$. Also in this case it immediately can be seen that to the solution, with the same $r_{i j}$-and $r_{j i}$-dependences as in equation (5.4), the same interpretation as before can be given. In accordance with the remark on this point in paragraph 3 the solution of equation (5.7) in the limit of $c$ to infinity will be the solution (5.4). So there is not much news in this alternative, but a wave equation with zero masses starting from:

$$
\left(E_{i j}^{2}+E_{j i}^{2}\right) \varphi_{i j}-c^{2}\left(\left(\widehat{p_{l j}}\right)^{2}-\left(\widehat{p_{j l}}\right)^{2}\right) \varphi_{i j}=0
$$

has a non constant solution in space and time coordinates. This is remarkable as a zero mass particle like a photon can result in a mass-like presence in open space. It may well be that this is the basis for the fact that in the Friedmann cosmological equations also energy related gravitational pull has to be adopted (Heacox, 2015). 


\section{Summary of the General Treatment and Further Steps to Be Taken}

In the preceding paragraphs some of the basic rules of Special Relativity theory and quantum mechanics are given insofar they are of relevance for developing a theory for the attractive force: gravitational attraction between two bodies. It is not specified what kind of bodies we are talking about and actually it is not even relevant. There is absolutely no speculation about the validity of these rules. They have shown their validity over and over, but working with both concepts should be done with care as the two concepts, although they have shown their validity, are not completely compatible. The energy equations according to Quantum Mechanics (3.5) and Special Relativity (5.3) show that the basic quantum equation has both linear and squared parameters whereas the relativistic one has only squares. Mathematically we say that the quantum equation is not co-variant and the relativistic one is co-variant. The consequence of co-variance is that the laws of physics are the same no matter the coordinate system, in which it is observed, is moving or not. With this latter determination in mind we can use both but we should be aware of the dangers involved.

When the two concepts were applied to particles with the aim to describe their movement and connected interaction, we have seen in two different ways that the movement of particles can be described for all the particles together, but individually, and also as in groups of two and only two members. These groups are then described together to arrive at a complete description of the movement of all groups together but mutually independent.

The grouping results in the description in a momentum based sub-space for the individual groups. In this momentum space the sub-spaces appear to be symmetric from the observer's point of view in $\mathrm{O}_{2}$, as shown in Figure 1. This symmetry will also be reflected in the symmetry of the gravitational interaction which will be shown in what follows.

It has been shown that particles, which can have high probability to be present in a physical entity like a solid material, or whatever, do have some small probability to show up in open space outside the entity where it normally is, or stated in quantum mechanical language: where it has highest probability. The probability to show up in open space can be so low that for normal practices it is of no relevance and in usual quantum mechanical considerations it is neglected. If we, however, want to come to a theory for gravity, we cannot ignore its probability in open space. This low probability already has the consequence that for individual particles the gravitational interaction is small but definitely not zero. The other consequence is that the extent where this probability is manifesting itself extends to the whole space. Gravity is a force that is present even at cosmological distances.

Now we come to the central transition point from quantum mechanics to quantum-based relativity.

The wave function as derived gives the presence of an entity to which a rest mass, $m_{0 i j}$, can be dedicated. In quantum mechanical language, as shown in paragraph 3 , this rest mass becomes an operator and therefore it has to be multiplied by the wave function and its conjugated function: $\psi_{i j}^{*} m_{0 i j}^{2} \psi_{i j}$ and we get:

$$
\psi_{i j}^{*} c^{4} m_{0 i j}^{2} \psi_{i j}=c^{4} m_{0 i j}^{2}\left(\frac{\alpha_{i j}}{r_{i j}}+\frac{\alpha_{j i}}{r_{j i}}\right)^{2} .
$$

This equation says that there is a probability that this pair potential can be found anywhere in the free space, but it is obviously concentrated between and around the two particles forming the pair. So there is a space coordinate dependent probability to find it somewhere, but this probability is not connected to other quantities like energy.

At this moment it is obvious already that $m_{0 i j}$ will be proportional to the masses of both participating particles in the interaction. But this is for the time being only a temporary conclusion. It will be justified later as it is of great importance for the final derivation of the gravity law.

Another very important thing is that from $\alpha_{i j} \beta_{j i} / m_{j}+\alpha_{j i} \beta_{i j} / m_{i}=0$ with $\alpha_{i j}=\alpha_{j i}$ it follows that there is for the $\alpha$-values some freedom in choosing its dependence on relativistic parameters such that the right hand side of equation (5.4) becomes an invariant as it should be, but also it is important for the conclusion that the form of the gravity law is independent of the mutually interacting masses of macroscopic bodies.

In figure 3 a comparison is made between the effect of two particles separately according to equation (5.1) and the same particles in the group as an entity according to equation (6.1). The overlap in the factor $\left(\alpha_{i j} / r_{i j}+\alpha_{j i} / r_{j i}\right)$ is due to an increased amplitude between the particles in the group. 


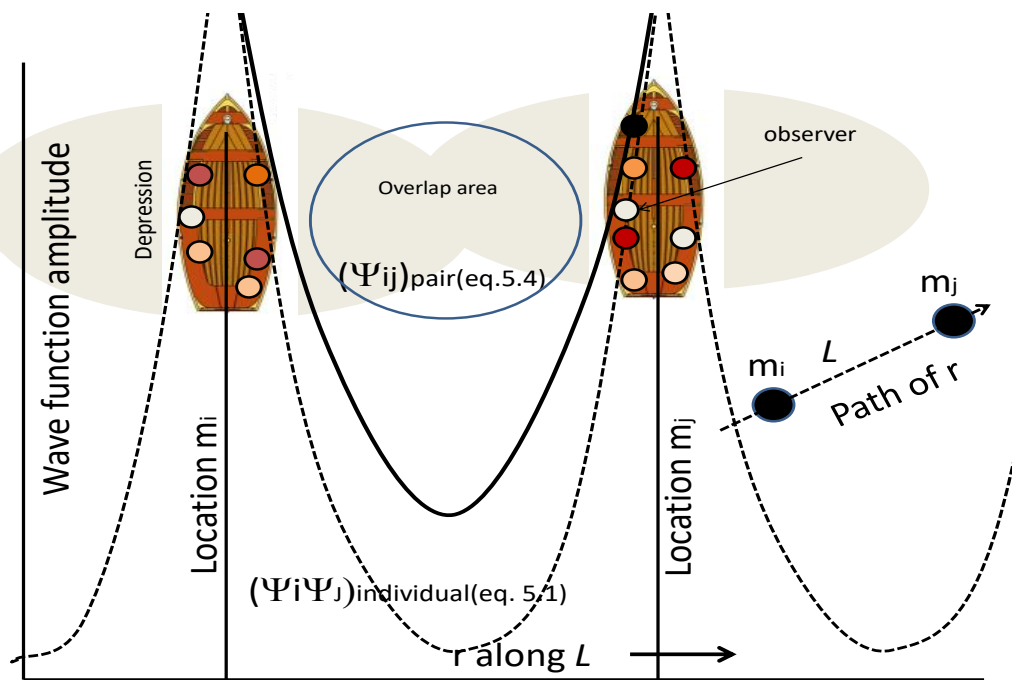

Figure 3: Overlap of amplitudes in a group of two particles in a group. An analogy is seen when two boats are sailing parallel to each other and create a depression in the water level. Due to the overlap in the depression between the boats there is a small attractive force between them.

\section{Relativistic Interaction}

Now, in the next step, the pair is considered as essentially one entity and the problem can be analyzed in the relativistic four dimensional space. We will draw up the KG-equation remembering the rules of adding up four-vectors and subsequently the formation of the invariant out of this sum. In this representation, however, the rest mass due to the interacting particles in the pair $(i j), m_{0 i j}$, is to be considered as an entity that is completely independent of all the other rest masses formed.

But the most important difference from the treatment before is that we will be entirely working in the momentum based sub-space $r_{i j}$ where the group is seen as one single entity. The energy reflects the energy of the two particles together as well as masses and momenta like: $\left.p^{2}=\widehat{\left(p_{l j}\right.}+\widehat{p_{\jmath l}}\right)^{2}$ and: $E^{2}=\left(E_{i}+E_{j}\right)^{2}$ with: $E^{2}-p^{2} c^{2}=m_{0}^{2} c^{4}$.

Again we will have to translate this equation into the appropriate quantum mechanical language for pairs as one entity and therefore make the following transformations:

$$
\begin{aligned}
& -p^{2} c^{2} \varphi_{i j, t} \varphi_{j i, t}=\left(m_{0}^{2} c^{4}-E^{2}\right) \varphi_{i j, t} \varphi_{j i, t}, \quad E^{2}=\left(\widehat{E_{l}}+\widehat{E_{J}}\right)^{2}=-\hbar^{2} \frac{\partial^{2}}{\partial t^{2}} \text { and: } \\
& \left.p^{2}=\widehat{\left(p_{l j}\right.}+\widehat{p_{j l}}\right)^{2}=-\hbar^{2}\left(\frac{1}{r_{i j}^{2}} \frac{\partial}{\partial r_{i j}} r_{i j}^{2} \frac{\partial}{\partial r_{i j}}+\frac{1}{r_{j i}^{2}} \frac{\partial}{\partial r_{j i}} r_{j i}^{2} \frac{\partial}{\partial r_{j i}}+\frac{\partial}{\partial r_{i j}} \frac{\partial}{\partial r_{j i}}+\frac{\partial}{\partial r_{j i}} \frac{\partial}{\partial r_{i j}}\right) .
\end{aligned}
$$

The last expression is, as different from earlier, a mixed sum of the momenta. This representation is a consequence of the fact that the particles have been treated only in pairs and that spherical symmetry remains to be adopted. The energy operators involving the momenta, which are used here, are given in equations (3.10a) and (3.10b).

Referring to Figure 2 the total relativistic KG-equation for a number of pairs $(\mathcal{N})$ now will be set up. There are $N$ particles which make a total of $\mathcal{N}=N ! / 2(N-2)$ ! pairs, each of which are described by a wave function as a solution of the initial Schrödinger equation. As before, the $\alpha$-values accommodate all necessary multiplication factors. Following the four-vector scheme of equation (2.3c), adding up for all pairs, treating them as mutually independent and taking into account the basic rules of quantum mechanics, lead to:

$$
\begin{aligned}
& c^{2} \hbar^{2} \sum_{i j}\left(\frac{1}{r_{i j}^{2}} \frac{\partial}{\partial r_{i j}} r_{i j}^{2} \frac{\partial}{\partial r_{i j}}+\frac{1}{r_{j i}^{2}} \frac{\partial}{\partial r_{j i}} r_{j i}^{2} \frac{\partial}{\partial r_{j i}}+\frac{\partial}{\partial r_{i j}} \frac{\partial}{\partial r_{j i}}+\frac{\partial}{\partial r_{j i}} \frac{\partial}{\partial r_{i j}}\right) \prod_{i j} \varphi_{j i, t} \varphi_{i j, t} \\
= & \sum_{i j} m_{0 i j}^{2}\left(\frac{\alpha_{i j}^{2}}{r_{i j}^{2}}+2 \frac{\alpha_{i j}}{r_{i j}} \frac{\alpha_{j i}}{r_{j i}}+\frac{\alpha_{j i}^{2}}{r_{j i}^{2}}\right) \prod_{i j} \varphi_{j i, t} \varphi_{i j, t}-\sum_{i j}\left(\widehat{E}_{l}+\widehat{E}_{j}\right)^{2} \prod_{i j} \varphi_{i j, t} \varphi_{j i, t}, \text { with: } \\
& \prod_{i j} \varphi_{i j, t} \varphi_{j i, t}=F(t) \prod_{i j} \varphi_{i j} \varphi_{j i}=\prod_{i j} \varphi_{i j} \varphi_{j i} \prod_{1}^{\mathcal{N}}\left(\frac{\alpha_{g}}{r_{g}}\right) e^{i\left(k_{g} r_{g}-\omega_{g} t\right)} .
\end{aligned}
$$


$m_{0 i j}$ is the rest mass to be dedicated to the interaction field created by the masses $m_{i}$ and $m_{j}$. This factor also accommodates the $c^{2}$ as in equation (6.1). The pairs in both products, in total $\mathcal{N}=N ! / 2(N-2) !$ are numbered by $g$, if there are $N$ particles. The term $e^{i\left(k_{g} r_{g}-\omega_{g} t\right)}$ expresses a wave propagating in radial direction representing the moving of individual groups, but with reducing amplitude, or, rather probability, as it progresses. If there is no interaction between members of the pairs $\left(\alpha_{m n}=0\right)$ we get the movement of the individual particles outside their local influence.

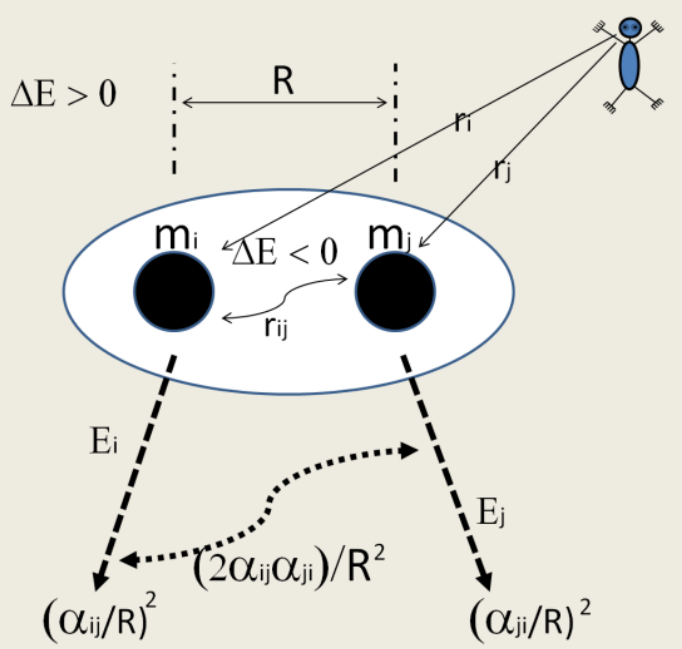

Figure 4: Energy transfer from the pair to the surroundings and the sub-space (white area) with internal exchanges as observed from far away.

This set-up has a very delicate interpretation. It shows that an observer from outside sees a pair creating a sub-space but cannot determine its structure inside. In the space inside, expressed by the coordinates $r_{i j}$ and $r_{j i}$, gravitational interactions are occurring. Our observer only sees the separate interacting members of the pair with an energy due to this interaction as is shown schematically in Figure 4. It is as if we see two persons who have made a secret agreement and are, by acting as a pair, exchanging information. We can see both persons but we cannot explain why they behave as they behave. This analogy will have some delicate interpretation to which we will come back in a footnote in paragraph $9(* * *))$.

As before, the time dependences can be removed by setting:

$$
\varphi_{i j, t} \varphi_{j i, t}=\varphi_{i j} \varphi_{j i} e^{i\left(E_{i j}+E_{j i}\right) t / \hbar},
$$

so that:

$$
\sum_{i j}\left(\widehat{E}_{l}+\widehat{E}_{j}\right)^{2} \varphi_{i j, t} \varphi_{j i, t}=\sum_{i j}\left(E_{i j}+E_{j i}\right)^{2} \varphi_{i j} \varphi_{j i}
$$

If all $\alpha^{\prime} s$ would have been equal to zero, a propagating wave $\varphi_{i j, t} \varphi_{j i, t}$ extending in the radial direction with the light velocity would have resulted. Non zero values of $\alpha$ reduce this speed and, as a consequence, give mass to the field $\varphi_{i j, t} \varphi_{j i, t}$. The proposed solution will be *):

$$
\varphi_{i j}=\gamma_{i j} r_{i j}^{-m_{0 i j} \alpha_{i j} / \hbar c}
$$

which is inserted into:

$$
\begin{gathered}
\sum_{i j}\left(E_{i j}+E_{j i}\right)^{2} \prod_{i j} \varphi_{j i} \varphi_{i j}-\sum_{i j} m_{0 i j}^{2}\left(\frac{\alpha_{i j}^{2}}{r_{i j}^{2}}+2 \frac{\alpha_{i j}}{r_{i j}} \frac{\alpha_{j i}}{r_{j i}}+\frac{\alpha_{j i}^{2}}{r_{j i}^{2}}\right) \prod_{i j} \varphi_{j i} \varphi_{i j}+ \\
+c^{2} \hbar^{2} \sum_{i j}\left(\frac{1}{r_{i j}^{2}} \frac{\partial}{\partial r_{i j}} r_{i j}^{2} \frac{\partial}{\partial r_{i j}}+\frac{1}{r_{j i}^{2}} \frac{\partial}{\partial r_{j i}} r_{j i}^{2} \frac{\partial}{\partial r_{j i}}+\frac{\partial}{\partial r_{i j}} \frac{\partial}{\partial r_{j i}}+\frac{\partial}{\partial r_{j i}} \frac{\partial}{\partial r_{i j}}\right) \prod_{i j} \varphi_{j i} \varphi_{i j}=0 .
\end{gathered}
$$

From the boundary condition that $\varphi_{i j}\left(r_{i j}, \alpha_{i j}\right)=0$ for $r_{i j}$ to infinity a fourth condition on the $\alpha^{\prime} s$ can be derived: $i v . \alpha_{i j}$ is positive under all circumstances.

Now some algebra needs to be done and it will be found that many terms on the left hand side are equal to the ones at the 
right hand side and therefore disappear **). We get:

$$
\left(E_{i j}^{2}+2 E_{i j} E_{j i}+E_{j i}^{2}\right) \varphi_{i j} \varphi_{j i}-c \hbar m_{0 i j}\left(\frac{\alpha_{i j}}{r_{i j}^{2}}+\frac{\alpha_{j i}}{r_{j i}^{2}}\right) \varphi_{j i} \varphi_{i j}=0 .
$$

At this point a remark has to be made: removing the term $\alpha_{k l}^{2} / r_{k l}^{2}$ means that some basic interaction occurs between the gravitational field and the particle. Obviously, for this separate term, a KG-equation can be formulated that shows that an entity with some relativistically derived mass operates and leaves behind a contribution to the interaction energy in the equation (7.6). So already at this point there is direct interaction between the pair and the field around. Also removing the term with $\alpha_{i j} \alpha_{j i} / r_{j i} r_{i j}$ means that there is a third interaction between the fields and the pair. It is schematically represented in Figure 4.

Taking all these interactions into account it is seen that all $\alpha$-terms, as they occur in equation (7.5), have disappeared. This has a profound meaning: in this model gravity is due to second order effects of the peculiarities of the spherical symmetry in a relativistic setting. The effect is weak and operates over a long range.

The contributions can now be redistributed, but first multiply all terms by $r_{i j} r_{j i}$ and observe that the proposed solution is the only one that gives a sharp value for the quantity $E_{i j} r_{i j}$ and $E_{j i} r_{j i}$ :

$$
\begin{gathered}
\left(E_{i j}^{2} r_{j i} r_{i j}+E_{i j} E_{j i} r_{j i} r_{i j}\right) \varphi_{i j} \varphi_{j i}-c \hbar m_{0 i j} \alpha_{i j} \frac{r_{j i}}{r_{i j}} \varphi_{i j} \varphi_{j i}=0, \\
\left(E_{j i}^{2} r_{j i} r_{i j}+E_{i j} E_{j i} r_{j i} r_{i j}\right) \varphi_{i j} \varphi_{j i}-c \hbar m_{0 i j} \alpha_{j i} \frac{r_{i j}}{r_{j i}} \varphi_{i j} \varphi_{j i}=0 .
\end{gathered}
$$

Cutting the equation (7.6) into two separate ones as given in equations (7.7a) and (7.7b) looks like arbitrary, as any cut between terms can be made. But if we now come back to the original suggestion, we see that the gravitational interaction becomes symmetric. The gravitational energy of particle $i$ is equal to the gravitational energy of particle $j$. It also reflects the point that a pair has to be seen as one entity. The observer cannot distinguish between the separate members of the pair.

*) The solution proposed, but more general: $\varphi_{i j}=\gamma_{i j} r_{i j}^{n}$, can also be applied to a KG-equation not involving pair formation so that $\varphi_{i}=\gamma_{i} r_{i}^{n}$ and giving a similar solution as $E_{i} r_{i}$. In the model the exponent is, according to point iii in paragraph 5 connected to pairs of particles so that in the present case it should be taken zero.

**) This solution (8.4) reduces all invariant and momentum terms in equation (7.5) but brings back a single gradient term. No other solution has better performance than the one proposed in (7.4) so it is to be considered as the most appropriate one. It is actually the spherical symmetry that is responsible for this remaining gradient term. In this model, therefore, the whole story is reduced to one simple statement: Gravity is a consequence of the three dimensional space with spherical symmetry and nothing else.

\section{Law of Gravity}

Most important for finding out how the members of a pair see each other is to look at the equations (7.7a) and (7.7b) by an observer at $m_{i}$ who sees the particle $m_{j}$ at a distance of $r_{i j}$ and an observer at $m_{j}$ who sees $m_{i}$ from a distance $r_{j i}$. They already know that $\alpha_{i j}=\alpha_{j i}=\alpha, r_{i j}=r_{j i}=R$, and $E=E_{i j}+E_{j i}$ with $E_{i j}=E_{i j}$ so that $E_{i j}=E / 2$. There are also no operators anymore in equation (7.7a) and (7.7b). In this conclusion a geometrical factor is established in momentum space as in Figure 1 which will need some more justification to be given later in this paragraph. The result is a simple relation:

$$
2(E / 2)^{2} R^{2}=2 E^{2}(R / 2)^{2}=c \hbar \alpha m_{0}
$$

The boundary condition in equation (7.2) is that $\varphi_{i j} \varphi_{j i}$ goes to zero for $\mathrm{r}$ to infinity so that $\alpha>0$, and because both particles in the pair change their energy by the same amount, it follows for the two members of the ensemble together that:

$$
E R=\sqrt{2 c \hbar \alpha m_{0}}
$$

and the gravitational force is given by $-\partial E / \partial R=$ constant $/ R^{2}$.

Now it is important to see how pairs consisting of particles of different masses present themselves in $\alpha$ and $m_{0}$. It looks like both parameters are tightly glued together in, for instance equation (6.1), but they stem from different places. $\alpha$ is derived from the quantum mechanical considerations whereas $m_{0}$ comes from the relativity theory. They have in common that both reflect the fact that pairs of obviously undefined mass units are responsible for the interaction. The most simple conclusion, which needs some justification, would be that: 


$$
m_{0 k l} \alpha_{k l}=\sigma_{1}\left(m_{k} m_{l}\right) \cdot \sigma_{2}\left(m_{k} m_{l}\right) \text {. }
$$

To justify this result there are two options following the four-vector addition-invariant rule of Special Relativity.

The first one is to add the four-vector components followed by forming the invariant. This procedure is shown in equation (2.3e). In this case the addition of the components of the four-vector, according the result of equation (8.2), will read like equation (8.3) for equal $2 c \hbar \alpha m_{0} / R_{i j}^{2}$-entities. This approach means that we consider all contributions together to $\sum_{i j} E_{i j}$ as one entity so that there is, for the four vector components which are added, one unique coordinate system and all $R_{i j}$-values are the same:

$$
\left(\sum_{i j} E_{i j}\right)^{2}=E_{\text {total }}^{2}=\left(\sum_{i j}\left(\sqrt{2 c \hbar \alpha m_{0}} / R_{i j}\right)\right)^{2}=f\left(m_{i}, m_{j}\right) 2 c \hbar \alpha m_{0} / R_{i j}^{2}
$$

In this view the two entities $m_{1}$ and $m_{2}$ are forming a single pair. We now build up two bodies $m_{l}$ and $m_{2}$ composed of $n_{l}$ and $n_{2}$ by their mass identical building blocks identified by $m_{i}$ in $m_{l}$ and $m_{j}$ in $m_{2}$, such that they in total make up the masses of $m_{l}$ and $m_{2}$. These building blocks can be anything like elementary particles, collection of atoms, as long as their masses are the same.

According to equation (8.2) the interaction between the two bodies $m_{1}$ and $m_{2}$ will be given by $(E R)_{12}=\sqrt{2 c \hbar \alpha_{12}^{c} m_{012}^{c}}$ in which the factor $\alpha_{12}^{c} m_{012}^{c}$ stems from the composite solution $\varphi_{12}^{c}$ derived from equation (7.4). This composite solution should be based on the $m_{0}$ - and $\alpha$-values of the whole entity $m_{l}$ and $m_{2}$ :

$$
\varphi_{12}^{c}=\gamma_{12} r_{12}^{-\left(\alpha_{12}^{c} m_{012}^{c}\right) / \hbar c}=\gamma_{12} r_{12}^{-\left(m_{012}^{c} / m_{0 i j}\right)\left(\alpha_{12}^{c} m_{0 i j}\right) / \hbar c} .
$$

First we consider the rest-mass carrying the gravitational interaction.

It is found that the pair ( $i j)$ has gravitational energy, say $\varepsilon$ and thus a mass $\varepsilon / c^{2}$. In the interaction space between $m_{l}$ and $m_{2}$ there are $n_{1} n_{2}$ pairs carrying the interaction between $m_{1}$ and $m_{2}$ and so we can conclude that $m_{012}^{c}=n_{1} n_{2} m_{0 i j}$. The function $\varphi_{i j}$ for the pair $(i j)$, will occur in $\varphi_{12}^{c} n_{1} n_{2}$ times and so we get for very small values of $\alpha_{i j} m_{0 i j} / \hbar c$ in the multiplication of $\varphi_{i j} \varphi_{j i}$ over all pairs between $m_{1}$ and $m_{2}: n_{1} n_{2} m_{0 i j}\left(\alpha_{i j}+\alpha_{j i}\right)=m_{0 i j}\left(\alpha_{12}^{c}+\alpha_{21}^{c}\right)$.

Combining the two arguments we finally get: $m_{012}^{c} \alpha_{12}^{c}=n_{1}^{2} n_{2}^{2} m_{0 i j} \alpha_{i j}$ and, remembering that $m_{1}$ and $m_{2}$ are composed of identical building blocks, we can conclude that $m_{012}^{c} \alpha_{12}^{c}=\sigma m_{1}^{2} m_{2}^{2}$, which leads to the result as in equation (8.3) with:

$$
E_{12}=E_{21}=\sqrt{2 \sigma c \hbar} \cdot\left(m_{1} m_{2}\right) / R \text {. }
$$

The second option is to take the $n_{1}$ and $n_{2}$ particles separately, form for each of them the invariant and add them all together. This procedures follows the scheme of equation (7.1a and b) and is according to the four-vector rule (2.3c). We then have to take the $\alpha m_{0}$ - values of the $n_{i}$ and $n_{j}$ entities separately so that we get:

$$
\sum_{i j} E_{i j}^{2} R_{i j}^{2}=n_{1} n_{2} E_{i j}^{2} R_{12}^{2}=2 c \hbar \alpha_{12}^{c} m_{0 i j}=n_{1} n_{2} 2 c \hbar \alpha_{i j} m_{0 i j},
$$

leading to:

$$
\mathrm{n}_{1}^{2} \mathrm{n}_{2}^{2} \mathrm{E}_{\mathrm{ij}}^{2} R_{12}^{2}=\left(E_{12} R_{12}\right)^{2}=\left(\sqrt{2 \sigma c \hbar} \cdot\left(m_{1} m_{2}\right)\right)^{2}
$$

In the Figure 5 this latter argument is visualized: all pairs that have been formed are acting independently so that we can add all the contributions of different masses at their individual locations together and in this way constituting bodies in the real world without any interference.

Finally we can conclude that, in view of this equation (8.5), also the gravitational interaction is proportional to both masses of the participating particles in the pair.

The distance between the masses is due to the $R_{i j^{-}}$values which, so far have been taken the same but this is not the case for larger entities. We therefore should add up all the interactions between particles, which in part see each other at different distances. This is a problem that has already been solved in the formulation of the classical theory of electrostatics (Panowsky, Philips, 1972). In this way, finally, Newton's gravitation law is obtained which, in vector notation reads: $\operatorname{div} \boldsymbol{g}=4 \pi \rho G$ in which $\boldsymbol{g}$ is defined as a gravitational field around an entity constituting a space coordinates dependent mass density $\rho . G$ is the well known gravitational constant equal to: $6.673 \times 10^{-11} \mathrm{~m}^{3} \mathrm{~kg}^{-1} \mathrm{sec}^{-2}$ (Heacox, 2015),

In accordance with the theory of electrostatics the gravity law can also be given in vector representation for bodies $M_{l}$ and $M_{2}$ which have their centers of gravity at a separation of $R$ : 


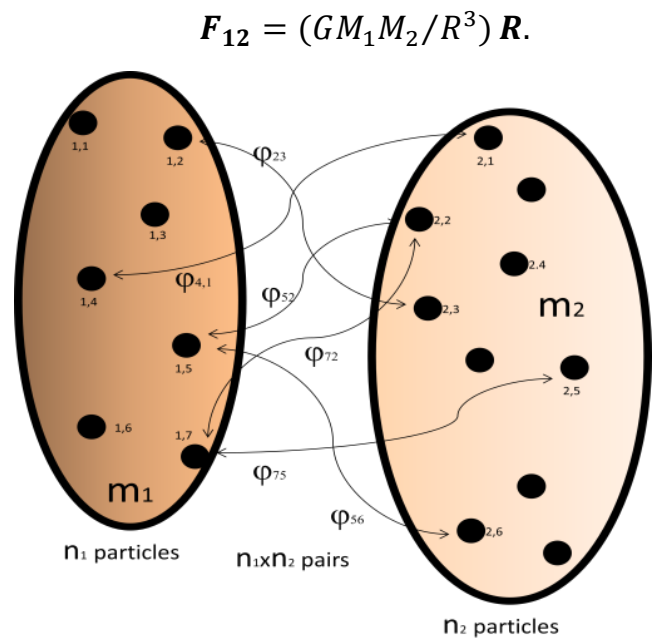

Figure 5: Interaction between masses by building up structures from equal mass units. In this graph the first number in the index of $\varphi$ refers to $m_{1}$ and the second to $m_{2}$.

From the equations (8.5) and (8.6) an explicit expression for the parameter $\sigma$ can be derived and also, with the help of these equations the small mass to be attributed to the gravitational interaction can be found. This $\sigma$ parameter is equal to $2.7 \times 10^{2} \mathrm{Jm} / \mathrm{kg}^{4}$.

In Figure 1 a sub-space is presented in momentum space with an observer in point $\mathrm{O}_{2}$ or, eventually, observers on the two particles. They see that the total energy of the pair $(i j)$ is lower than the energy of the particles separately. But since $E_{i j}=E_{j i}$ they must conclude that $r_{i j}=r_{j i}$. The locations where $r_{i j}=r_{j i}$, have in this respect, a specific meaning.

If we form the operator $\widehat{p_{l j}} \pm \widehat{p_{j l}}$ and apply it to the wave function $\psi_{i j}=\left(\frac{\alpha_{i j}}{r_{i j}}+\frac{\alpha_{j i}}{r_{j i}}\right) e^{i \beta_{i j} r_{i j}+i \beta_{j i} r_{j i}}$ we get the "eigenvalue" equation:

$$
\frac{\hbar}{i}\left(\frac{\partial}{\partial r_{i j}} \pm \frac{\partial}{\partial r_{j i}}\right) \psi_{i j}=\left\{\hbar\left(\frac{\alpha_{i j} \beta_{i j}}{r_{i j}} \pm \frac{\alpha_{j i} \beta_{j i}}{r_{j i}}\right)-\frac{\hbar}{i}\left(\frac{\alpha_{i j}}{r_{i j}^{2}} \pm \frac{\alpha_{j i}}{r_{j i}^{2}}\right)\right\} e^{i \beta_{i j} r_{i j}+i \beta_{j i} r_{j i}}
$$

In the earlier argument as shown in Figure 5 we have the mass units $m_{1}=m_{2}$, or for this argument $m_{i}=m_{j}$, and so we have $\beta_{i j}=-\beta_{j i}$, and we get a pure "eigenvalue" equation for the operator $\widehat{p_{l j}}-\widehat{p_{\jmath l}}$ if $r_{i j}=r_{j i}$. So the observer in point $\mathrm{O}_{2}$ in Figure 1 with $r_{i j}=r_{j i}=R / 2$ will see a sharp and well defined value for the difference between the momenta of the mass entities $m_{i}$ and $m_{j}$. With the same properties for $\beta$ the expectation value of the operator $\widehat{p_{l j}}+$ $\widehat{p_{\jmath l}}$ will be zero. Once again it follows that the momenta of the masses in the pair will be opposite but equal in absolute value.

The observer can evaluate equations (7.7a) and (7.7b) but in that case with $E=E_{i j}=E_{j i}$ leading to the same result as equation (8.1):

$$
2(E / 2)^{2} R^{2}=2 E^{2}(R / 2)^{2}=c \hbar \alpha m_{0} .
$$

The other option is two observers, one on $m_{i}$ and one on $m_{j}$, so that $r_{i j}=r_{j i}=R$, but now with $E=E_{i j}+E_{j i}$ and the result of equation (8.8) or (8.1). 


\section{Transfer of Energy and Mass}

In the analysis going from equation (7.5) to (7.7) terms are disappearing due to the solution proposed in equation (7.4). But this has to be interpreted with caution. The pair function $\varphi_{i j} \varphi_{j i}$. in equation (7.1) represents a field carrying the gravitational energy. Therefore, the disappearance of the generator at the left hand side of equation (7.5), $\left(\alpha_{i j} / r_{i j}+\alpha_{j i} / r_{j i}\right)^{2}$, involves exchange of energy from the pair to the surrounding space which is equal to the energy given in equation (8.2). As a consequence, when the positive value for the energy is taken, the energy of the pair itself is reduced by the same amount. In that case the interaction between the members of the pair is attractive. The process is schematically shown in Figure 4. The transferred energy is the difference between the energy levels shown Figure 6.

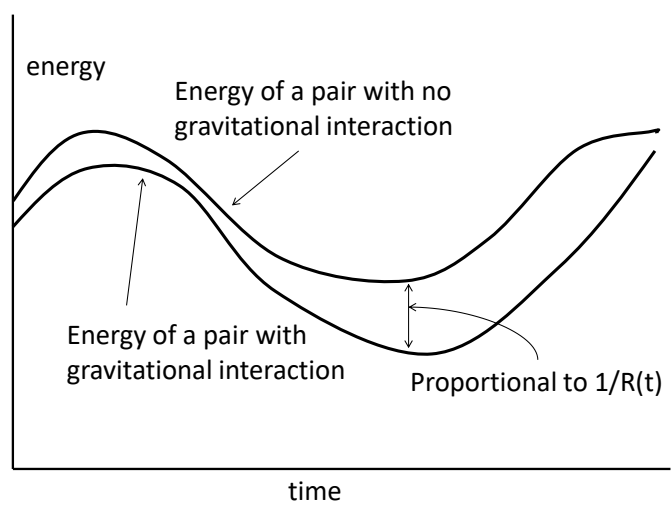

Figure 6. Energy difference between local states and incorporating gravitational interaction.

The opposite situation in which the energy of the pair is positive, which in principle is allowed by the Einstein energy equation (2.4), is not possible when we assume that the energy of the vacuum, to be taken as the reference point, is zero.

In this interpretation the interaction between mass and the surroundings is a means to transfer mass related energy $\left(m c^{2}\right)$ to gravitational energy. This transfer changes the rest masses of the pair but does not create new mass. The consequences at a larger scale are worked later in this paragraph.

If, however, the vacuum state is, as it is generally believed, a non-zero energy state there might be energy available which increases with the interaction area, the white area in Figure 4, that can be transferred to the pair.

The situation could be such that, when the distance between the members of the pair increases, the energy needed is reducing whereas the energy, or number of fluctuations carrying sufficient energy is increasing. It means that at some separation distance of the members of the pair the interaction can become repulsive as the Einstein equation allows both negative and positive values for the interaction energy.

A solution for the Schrödinger equation of a pair of particles for an observer at distances $r_{i j}$ and $r_{j i}$ from particle $i$ and $j$ is given in equation (5.4). Now if we put our observer close by particle $i$, the second term in equation (5.4) becomes negligible against the first term:

$$
\psi_{i j}=\left(\frac{\alpha_{i j}}{r_{i j}}+\frac{\alpha_{j i}}{r_{j i}}\right) e^{i \beta_{i j} r_{i j}+i \beta_{j i} r_{j i}} \cong\left(\frac{\alpha_{i j}}{r_{i j}}\right) e^{i \beta_{i j} r_{i j}+i \beta_{j i} r_{j i}} \text { and: } \psi_{i j}^{*} \psi_{i j} \cong\left(\frac{\alpha_{i j}}{r_{i j}}\right)^{2}
$$

The KG-equation in operator language now reads $* * *)$ :

$$
-\hbar^{2}\left(\frac{\partial^{2}}{\partial t^{2}}-c^{2} \frac{1}{r_{i j}^{2}} \frac{\partial}{\partial r_{i j}} r_{i j}^{2} \frac{\partial}{\partial r_{i j}}\right) \varphi_{i j, t}=m_{0 i j}^{2}\left(\frac{\alpha_{i j}}{r_{i j}}\right)^{2} \varphi_{i j, t} .
$$

Setting the right hand side to zero, a mass-less particle, we see an equation for a travelling wave at the speed of light. To get rid of the singularity we set $\alpha_{i j} / r_{i j}=\alpha_{i j} / r_{i p}$ for $r_{i j}<r_{i p}\left(=r_{p}\right)$, and removing the first term on the left hand side gives the London Equation that explains the shielding of the inside of a superconducting material from the outside magnetic field: the "Meissner" effect (Bardeen, et.al., 1957). A similar thing can be imagined in this case with the $\varphi_{i j, t}$-field for $r_{i j}<r_{p}$. The distance $r_{p}$ can be identified as the distance from the centre to where local influences have no impact.

We can solve the equation (9.2) with in the right hand term $r_{p}$ for $r_{i j}$, but it is not necessary as it can immediately be 
seen that it dedicates mass to the field in the vicinity of the particle which is equal to $m_{p}=m_{0 i j} \alpha_{i j} / r_{p} \mathrm{c}^{2}$. As this is the mass to be attributed to the $i^{\text {th }}$ particle, due to another particle somewhere in the surroundings, we will have to add up over all particles which can make a pair with our particle, so with $\left.m_{p}=m_{i} * * *\right)$ :

$$
m_{p}=\sum_{j} m_{0 i j} \alpha_{i j} / r_{p} c^{2}=\left(\sigma / r_{p} c^{2}\right) \sum_{j} m_{p}^{2} m_{j}^{2}
$$

The consequence is that either $m_{p}=0$, a mass-free particle, or $m_{p}=r_{p} c^{2} / \sigma \sum_{j} m_{j}^{2}$, with, as shown, $m_{0 i j} \alpha_{i j}=$ $\sigma m_{i}^{2} m_{j}^{2}$. First the equation allows that there are mass-free particles like photons which make no pairs according the theorem based on the Schrödinger equation, but it can, according to the KG-equations in paragraph 5, equation (5.8). It could generate gravity as it is argued in Chapter 9: Cosmography of W.D. Heacox's book on the expanding Universe. Second, the other solution is that there is a mass carrying particle whose mass becomes higher when $r_{p}$ increases and, most important, it is all the mass in the surroundings that generate the mass of the $i^{\text {th }}$ particle. It is actually mass due to the field, but since the singularity moves with the particle the observer nearby can only interpret it as a mass contribution to the particle he is looking at. The conclusion taken here corresponds to Mach's ideas about the effect of all physical entities in the universe.

It would be tempting to evaluate $m_{p}$ but, as we know already from observation, it is better to estimate the size or the extension of the particle if only this effect is responsible for the mass. The analysis concerns incredibly large and small numbers but leads to a surprising outcome.

Starting from $m_{p}=r_{p} c^{2} / \sigma \sum_{j} m_{j}^{2}$ and assuming that the mass of the universe is basically due to protons and neutrons with almost the same mass, so $m_{p}=m_{j}$, and assuming there are $N$ particles in the whole universe giving it a total mass of $M_{u}$ we can set:

$$
M_{u}=N m_{j}=N r_{p} c^{2} / \sigma \sum_{j} m_{j}^{2}=N r_{p} c^{2} / \sigma N m_{j}^{2}=r_{p} c^{2} / \sigma m_{j}^{2} .
$$

Estimates of the size of the universe on the basis of the inverse Hubble constant and the fact that the average intergalactic density is 1000 hydrogen atoms per cubic meter tells us that the total mass of the universe is of the order of $10^{55} \mathrm{~kg}$. $\sigma$ is calculated in paragraph 8 at $2.7 \times 10^{2} \mathrm{Jm} / \mathrm{kg}^{4}$ and the proton mass is $1.7 \times 10^{-27} \mathrm{~kg}$, Wikipedia.org/proton. It leads to an estimate for the $r_{p}$-value in the order of $10^{-15} \mathrm{~m}$, which is about the size of a proton: 0.8 femtometers (Antognini, et.al., 2013). An electron which is 1840 times lighter than the proton will, according to equation (9.3), see the same surrounding as the proton, so its size would be smaller by the same factor.

Although the correspondence with measured data is surprisingly good, it is still a rough estimate and not without speculation.

Even a discrepancy by a factor of 10 would already be acceptable for the outcome of this analysis. For instance, the sub-space due to the generator $m_{0 i j}^{2}\left(\alpha_{i j} / r_{i j}+\alpha_{j i} / r_{j i}\right)^{2}$ would be a quantum-mechanical reality, but it says nothing about its internal structure and interactions. The mass of the universe is rather uncertain in view of the discussion about dark matter, and the proton size, or how to define it, is not so obvious.

***) The observer on particle $m_{i}$ sees the effect of the particle $m_{j}$ in its surroundings and coordinate system at the distance $r_{j i}$ and vice versa. This means that, formally, the operators $\widehat{p_{l j}}, \widehat{p_{j l}}$ work on the coordinates $r_{j i}, r_{i j}$ and therewith define the coordinate dependences on $r_{i j}$ and $r_{j i}$. So there are as many coordinate systems as there are particles, or mass carrying entities. The appreciation of this "kind of secret" code allows us to interpret gravity and mass dedication as two manifestations of one and the same mechanism. But in this latter respect equation (9.3) should be interpreted with caution. For instance, if we would squeeze $n$ particles in one the same coordinate system, we would see that the volume expands from $4 \pi r_{p}^{3} / 3$ to $4 \pi n^{3} r_{p}^{3} / 3$, whereas if we cluster these $n$ particles together the volume increases to $4 \pi n r_{p}^{3} / 3$.

\section{The Bohr-Einstein Controversy}

The model describing the gravitational interaction between particles has a some relation with the classical Bohr-Einstein dispute, Bohr-Einstein Debates, (Wikipedia.org). This dispute has been dealt with in many sessions between 1913 and 1930 as a subject of the Solvay Conferences. The issue was Einstein's belief that the quantum theory is an incomplete theory as he rejected the idea that positions in space-time could never be completely known. Einstein did not want to allow the uncertainty principle to necessitate an apparently random non-deterministic mechanism by which the law of physics would be operating.

The controversy culminated in the well known Einstein-Podolsky-Rosen Paradox, (EPR) of 1935 which comes close to the ideas presented in this document. Two particles have a common source, like two photons originating from one process in terms of space and time. From a quantum mechanical point of view the set of the two particles are represented by a joint wave function. One particle has orientation up (U) and the other down (D) and we do not know 
on beforehand which of the two is up and which or down:

$$
\psi_{12}=\psi_{1}(U) \psi_{2}(D)+\psi_{1}(D) \psi_{2}(U) .
$$

This is a superposition of two states of the ensemble. At some moment we do an experiment and find out that one of the particles is specified as "up". From quantum theory we conclude that the other should be "down". It might be that the system is influenced by the measurement so that the result "up" emerged, but the other particle is definitely not influenced and we know that it is characterized as "down". It appears that the wave function has selected the option $\psi_{1}(U) \psi_{2}(D)$ out of the superposition. From quantum mechanical point of view the process occurs independently of where in space and at which moment it takes place.

For Einstein this was unacceptable and he suggested that the particle might have some "hidden variables" which we do not know and which decide the choice of the system. Niels Bohr could, however, justify his result by working out the situation in a more statistical way as quantum mechanics is basically a theory of probabilities which has been experimentally confirmed on several occasions (Aspect, et.al., 1982).

We can now identify the solution for the pair potential in equation (5.4) or, more generally, equation (5.6), for a multi group particle pair system, in a similar way as the "up/down" combination given above. As Max Born pointed out in a letter to Einstein (Einstein et.al., 1935): "There is a wholeness to a quantum events that persists over time and space and makes linkages possible". These linkages, leading to the definition of the invariant in the KG-equation, apparently, give rise to the gravitational interaction. Apparently a single particle sees an environment and makes pairs with all of the particles around it. Suppose that at the other side of our galaxy two particles $k, l$ annihilate. Suddenly the number of pairs reduces and this is seen by our particle. This change in the wave function:

$$
\prod_{i j} m_{o i j}\left(\frac{\alpha_{i j}}{r_{i j}}+\frac{\alpha_{j i}}{r_{j i}}\right) e^{i \beta_{i j} r_{i j}+i \beta_{j i} r_{j i}} e^{i\left(E_{i j}+E_{j i}\right) t / \hbar} \text { to: }\left\{\prod_{i j} m_{o i j}\left(\frac{\alpha_{i j}}{r_{i j}}+\frac{\alpha_{j i}}{r_{j i}}\right) e^{i \beta_{i j} r_{i j}+i \beta_{j i} r_{j i}} e^{i\left(E_{i j}+E_{j i}\right) t / \hbar}\right\}_{i, j \neq k, l}
$$

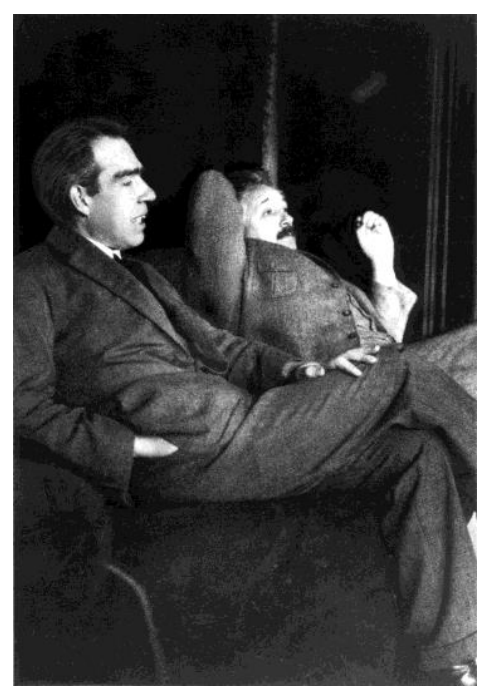

Figure 7. Niels Bohr and Albert Einstein in Ehrenfest's home in Leiden in December 1925.

produces a gravitational wave travelling through empty space at the speed of light and that adjusts to the new situation. But the information that the gravitational wave must start has already been exchanged between our particle with the observer and the annihilating pair. Again we end up in the same controversy as between Bohr and Einstein.

\section{Summary of Preceding Paragraphs and Some Remarks for Completeness}

An important first conclusion in an earlier summary was that particles with a mass can be described as a single non-interaction pair containing only two members. The individual members can make pairs with all other mass in its surroundings. This, already peculiar pair effect, is used in the KG-equation which, in a quantum-mechanical representation, describes a field around these members. The second conclusion made is that energy is subtracted from the pair and gives rise to an attractive force between the two members of a pair. By setting this force equal to the well-known parameters of Newton's gravity law, numerical values can be given to the main parameters found with the KG-equation. It is then found that an observer watching the pair will see that the pair has two members, but he cannot see how they interact or exchange information, only that it leads to a force between them. This force is independent of 
the movement of the members in the sub-space as shown in Figure 1.

The field that occurs due to the KG-equation is not only present outside the particle but must also have its influence in areas where the particle mass density manifests itself. Not much is known about what this field inside the particle looks like and its local interactions, but the most simple approach would be to assume that the amplitude of the generator of this field is constant. The dependence on space coordinates of this field inside the outer boundary of the particle leads to the attribution of mass. This mass is then found to be a consequence of all the interactions which the single particle has with the surrounding mass in which the distance, apparently, plays no role. If we start from the values of the parameters derived from the gravitational interaction, and the known mass of a proton, its outer boundaries can be calculated which agree surprisingly well with the data found experimentally. However, it must be stressed that this last reasoning is speculative.

One point, difficult to accept from logical point of view, is that members of a pair seem to have instantaneous contact no matter how far they are apart and therewith generate the interaction field that gives rise to gravity. Gravity waves move at the speed of light or slightly less, depending on the mass density it is moving through, but its generator works apparently without delay. The situation is the same as the classical debate in the previous century between Einstein and Bohr and have remained to be an issue which is hard to believe but more than once shown to be true.

Another point to remark here is the occurrence of a generator creating a sub-space. It follows unambiguously from the Schrödinger equation but nothing can be said about its internal structure where particles are entangled and apparently exchange information. This might be close by the idea of Einstein about "hidden variables".

The gravitational constant in Newton's law, $G$, is expressed by $G=\sqrt{2 \sigma c \hbar}$ in which the parameter $\sigma$, equal to $2.7 \times 10^{2}$ $\mathrm{Jm} / \mathrm{kg}^{4}$, can be seen as a universal constant that connects relativity with quantum mechanics.

The surprising, and at the same time bizarre, conclusion of the analysis given is that, apparently, each single particle has interaction with all other particles in the cosmos. It means that in the universe an unimaginable number of pair-wise interactions exists with greatly varying intensity and extensions and which depend on the masses of the members of the pair. It is difficult to comprehend, but it follows unambiguously from the equations describing the behaviour of the pairs.

As a last remark for this paragraph, causality is of importance to keep in mind. The model starts from the fact that there are masses, and it is seen that they can form pairs and generate gravity. It yields numerical data about the masses following gravitational parameters. The strength of the model is the consistency of the data with what we observe in reality. On the other hand one can say that the mass can be introduced into the Schrödinger equation as an unknown quantity and the theory comes back with a numerical value for it if the size of the particle is known.

\section{Gravity Depending on Dynamical Mass}

In paragraph 4 at the end it is mentioned that a group as a whole, identified with the label $g$, has kinetic energy and therefore a relativistic mass equal to $\gamma_{g} m_{g}$. Although the present theory is only concerned about the situation in the sub-space $r_{i j}$ where gravity originates, it still is of interest to know the dynamic mass of the group because of the fact that the Hamiltonian operator has been modified. The momentum of the group, $\widehat{p_{g}} / \sqrt{2 m_{g}}$, will determine the dynamic mass to be dedicated to the members of the group. To see this we come back to equation (8.5), now renumbered to (12.1):

$$
E_{i j}=\sqrt{2 \sigma c \hbar} \cdot\left(m_{i} m_{j}\right) / R,
$$

This equation is valid for the sub-space in which the gravitational energy is independent of the momenta of the particles in the group. But by inspection we see a problem:

For an observer outside the sub-space the right hand side in equation (7.5) should be invariant under Lorentz transformation. However, the $r_{k l}$ transforms as a member of a four-vector. Therefore, the parameters $\alpha_{k l}$ or, in the case of equation (12.1), $\sigma$ should transform in the same way as $r_{k l}$, but apparently it would make left and right hand side in equation (8.2) transform differently, which cannot be the case. We should, however, notice that the Planck's constant, $h$, is invariant, but $\hbar=h / 2 \pi$ is not.

Make the following "thought-experiment". Consider a pair flying away from us at a speed $v$ such that the separation vector of the members of the pair is aligned in the direction of $v$. Due to the fact that $\pi$ transforms just like $1 / r_{k l}$ the result is that the interaction energy of the pair we measure becomes invariant. There is invariance throughout if the alignment perpendicular to the speed. So the conclusion is that the interaction energy in the pair is invariant and independent of the alignment towards the observer. We can see the pair moving by and, whatever alignment they have, we will see the same interaction energy. But for the observer outside the sub-space, actually in point $\mathrm{O}_{1}$ in figure 1 , the group as a whole is moving which gives a dynamical mass to the particles in the group, but with the same $\gamma_{g}$ - factor. 
Knowing this we can from equations (4.5a) and (4.5b), in principle, give the value for this group momentum if the replacement of the vectors $\widehat{p_{k}} / \sqrt{2 m_{k}}$ by their relativistic equivalents has been done. However, there remains a disturbing $\cos \delta_{1}$-term making a general solution inappropriate. But the purpose of an endeavour in which such a group related dynamic mass is significant makes only sense where gravity is important and speeds are approaching the speed of light. So it is not relevant outside the realm of cosmology.

In this respect the main problem of the incompatibility between quantum theory and relativity comes to the surface. We therefore have to carefully replace the vectors in Figure 1 by the relativistically relevant ones which are to be derived from the equations (4.5a) and (4.5b) leading to the transitions:

$p_{a} / \sqrt{2 m_{a}} \mapsto c m_{a} \sqrt{\gamma_{a}^{2}-1}$ with $a=i, j$ and $g$.

Now we can put our observer on one of the interacting particles, say $m_{i}$ in the group ( $\left.i j\right)$, and consider the surroundings from this point of view so that $p_{i}=0$. In this case $\cos \delta_{1}=-1$, but because $p_{i}=0$ the $\cos \delta_{1}$ - factor has no influence anymore. We end up in a rather complicated situation if we want to know the mass and $\gamma_{g}$ - values for the group and we find non relativistic:

$$
v_{g}^{2} / c^{2}=\frac{m_{j}}{2\left(m_{i}+m_{j}\right)} v_{j}^{2} / c^{2}, \text { and relativistic: } \gamma_{g}^{2}-1=\frac{m_{j}^{2}}{2 \eta^{2}\left(m_{i}+m_{j}\right)^{2}}\left(\gamma_{j}^{2}-1\right)
$$

The extra parameter $\eta$ complicates the situation. If our observer is on mass $m_{i}$ which is much smaller than $m_{j}: \eta=1$, and both equations are identical. But in paragraph 8 , equation (8.5) we have constructed our bodies with building blocks of masses $m_{i}$ which all are identical in their masses. We should, therefore, start from the case of $m_{i}=m_{j}$, so that: $\eta=\sqrt{1 / 2}$. The result is:

$$
\gamma_{g}{ }^{2}-1=\left(\gamma_{j}^{2}-1\right) / 4
$$

With the aid of the definition of $\gamma$ it is easily changed into the relation:

$$
v_{g}^{2} / c^{2}=v_{j}^{2} /\left(4 c^{2}-3 v^{2}\right) \text {. }
$$

This gives the mass to be allotted to both members of the group. At low velocities $(\mathrm{v}<<\mathrm{c})$, The mass of the group particles is determined by half the speed of the moving particle. When the speed of the moving particle approaches the light velocity, both speeds become equal. This result is similar to the velocity addition rule for relativistic velocities on the basis of standard relativity theory (Ney, 1965), but in this case arrived at in way involving gravity.

At low speeds we have to dedicate dynamic mass to both particles and the equation will read:

$$
\boldsymbol{F}_{\mathbf{1 2}}=\boldsymbol{R} G\left(M_{01} / \sqrt{\left(1-v^{2} / 4 c^{2}\right.} M_{02} / \sqrt{\left(1-v^{2} / 4 c^{2}\right.}\right) / R^{3} .
$$

When speeds are approaching the speed of light, of course, the speeds of both particles are still the same and opposite, but at the value $v_{j}$. An alternative way of interpreting equation (12.5) is to place the observer in the sub-space in the middle between the two particles so that the observer sees their speeds $v^{\prime}=v / 2$ and opposite and the distance $R^{\prime}=R / 2$. In that case the equation becomes:

$$
\boldsymbol{F}_{12}=\boldsymbol{R}^{\prime} G\left(M_{01} / \sqrt{\left(1-v^{\prime 2} / c^{2}\right.} M_{02} / \sqrt{\left(1-v^{\prime 2} / c^{2}\right.}\right) / 4 R^{\prime 3} .
$$

This interpretation has to be considered as an alternative interpretation of equation (12.5) and not of the real situation of two particles moving away from the observer at equal but opposite speeds. This is because the equations are derived for the case that we have taken the momentum of one of the group members as zero. It, however, allows a remarkable interpretation. It looks like a "mirror" mass shows up at a distance of $\mathrm{R}$ from the moving mass that moves at the same speed as the moving one. Far from the light speed the relative speed between the two masses is double the speed seen by the observer but when it approaches c, the relative speed becomes c as well.

In conclusion it can be said that particles in a group in the sub-space have gravitational interaction have masses which must be corrected with the relativistic transformation factor $\gamma_{g}$ as defined by equation (12.2a) and (12.2b) with the right value for the parameter $\eta$.

The kinetic energy of the group remains to be defined by the value:

$$
E_{k i n}=T_{k}=M_{01}\left(\gamma_{1}-1\right) c^{2}+M_{02}\left(\gamma_{2}-1\right) c^{2} .
$$

The allocation of dynamics masses to the particles as members of a group of two solves the problem that is encountered many times by taking the dynamic values for the masses in equation (8.6): Newton's law. The problem that shows up by this allocation is that the gravitational potential energy becomes dependent on the location of the observer towards the two masses. This problem does not occur in the analysis as presented here. 


\section{Discussion and Conclusions}

An attempt is made to find an explanation for Newton's gravity law, starting from well established and proven theorems: Special Relativity and Quantum Mechanics. Although these two theories cannot be readily combined, it is possible to use the outcome of quantum mechanical considerations as starting point for further analysis by taking into account the rules of Specific Relativity. If we apply the two concepts in those areas where they have their applicability it was proved possible to derive the gravity law as it has been established already more than three hundred years ago. The main issues in the analysis are:

1. We can separate the local behavior of particles in its direct environment like a gas, liquid or a solid from its behavior in free space as a member of a larger entity.

2. We can modify the Hamiltonian of a set of two individual particles, or tightly connected entities, such that for two and only two of such entities, characterized by their masses, the Hamiltonian is represented by a group kinetic energy operator and a second part which is the direct interaction in a separate momentum based sub-space.

3. From this, a group wave function and a wave function representing the members in the group emerge, the first one is found to be responsible for the dynamic masses to be allotted to the particles in the gravity law and the second one is responsible for the gravitational interaction.

4. The two particle wave function is then recognized as a pair potential in a sub-space between the members of the group and is taken as the relativistically invariant rest mass in the Klein Gordon field equation.

5. By solving the Klein-Gordon field equation for the pair represented as a single entity we finally arrive at the right form of Newton's law of gravity. Also by adding up the basic functions for single group of particles, or groups of particles, the right form of the gravity law between large bodies is obtained.

6. Considering the dynamics of the group as a whole also the influence of the dynamic relativistic mass in the gravity equation is derived.

7. The Klein-Gordon equation is also found to be applicable at the level of a single particle and gives a value for its mass in dependence of all mass around in the entire universe. Most surprising is that the calculated values are of the right order even though the numbers that are going into the equations are extremely large and extremely small.

The model proposed in this document is a particle-based model in which first a Schrödinger equation is solved for a pair of mass-based entities. This solution is then taken into the KG-equation which comes with an attractive force between the particles in the pair. By taking the experimentally derived attractive force between two masses, the parameters can be given a numerical value.

The model does not directly lead to the concept of curvature of space-time as it follows from the General Relativity theory. This does not mean that the present theory violates General Relativity. General Relativity starts from the concept of curved space-time and leads to Newton's gravity law. It describes the behavior of matter as a movement in a four dimensional curved space. The present theory starts from quantum mechanical concepts combined with Specific Relativity and arrives at Newton's law as well. One can say that Newton's law has united the microscopic and macroscopic ideas. 


\section{References}

Antognini, A., \& et. al. (2013). Science 339, 417-420. https://doi.org/10.1126/science.1230016

Aspect, A., Grangier, P., \& Roger, G. (1982). Experimental Realization of Einstein-Podolsky-Rosen-Bohm Gedankenexperiment: A New Violation of Bell's Inequalities, Physical Review Letters, 49(2), 91-94.

Bardeen, J., Cooper, L. N., \& Schrieffer, J. R. (1957): Theory of Superconductivity: Physical Review $108,1175$. https://doi.org/10.1103/PhysRev.108.1175

Bohr-Einstein Debates: Wikipedia: https://en.wikipedia.org/wiki/Bohr\%E2\%80\%93Einstein_debates.

Born, M. (1924). Einstein's Theory of Relativity, London, Methuen.

Einstein, A. (1923). Four Lectures at Princeton University in 1921. Princeton University Press, 119. "Can Quantum-Mechanical Description of Physical Reality Be Considered Complete?"

Einstein, A. Podolsky, B., \& Rosen, N. (1935). Physical Review Letters, 47. 777.

Feynman, R. (1965). Lectures on Physics, Volume II.

Heacox, W. D. (2015). The Expanding Universe, Cambridge University Press.

Heitler, W. (1945). Elementary Wave Mechanics, Oxford Clarendon Press.

Messiah, A. Quantum Mechanics Vol. 1, North Holland Publishing Company, Amsterdam.

Messiah. A. (1985). Quanten-Mechanik, Band 2, Walter de Gruyter, Berlin.

Ney, E. P. (1965). Electromagnetism and Relativity: Harper \& Row, New York.

Panowsky, W., \& Philips, M. (1972). Classical Electricity and Magnetism, Addison Wesly Publishing Company.

Ryder, L. (2009). Introduction to General Relativity, Cambridge University Press. https://doi.org/10.1017/CBO9780511809033

\section{Symbols used}

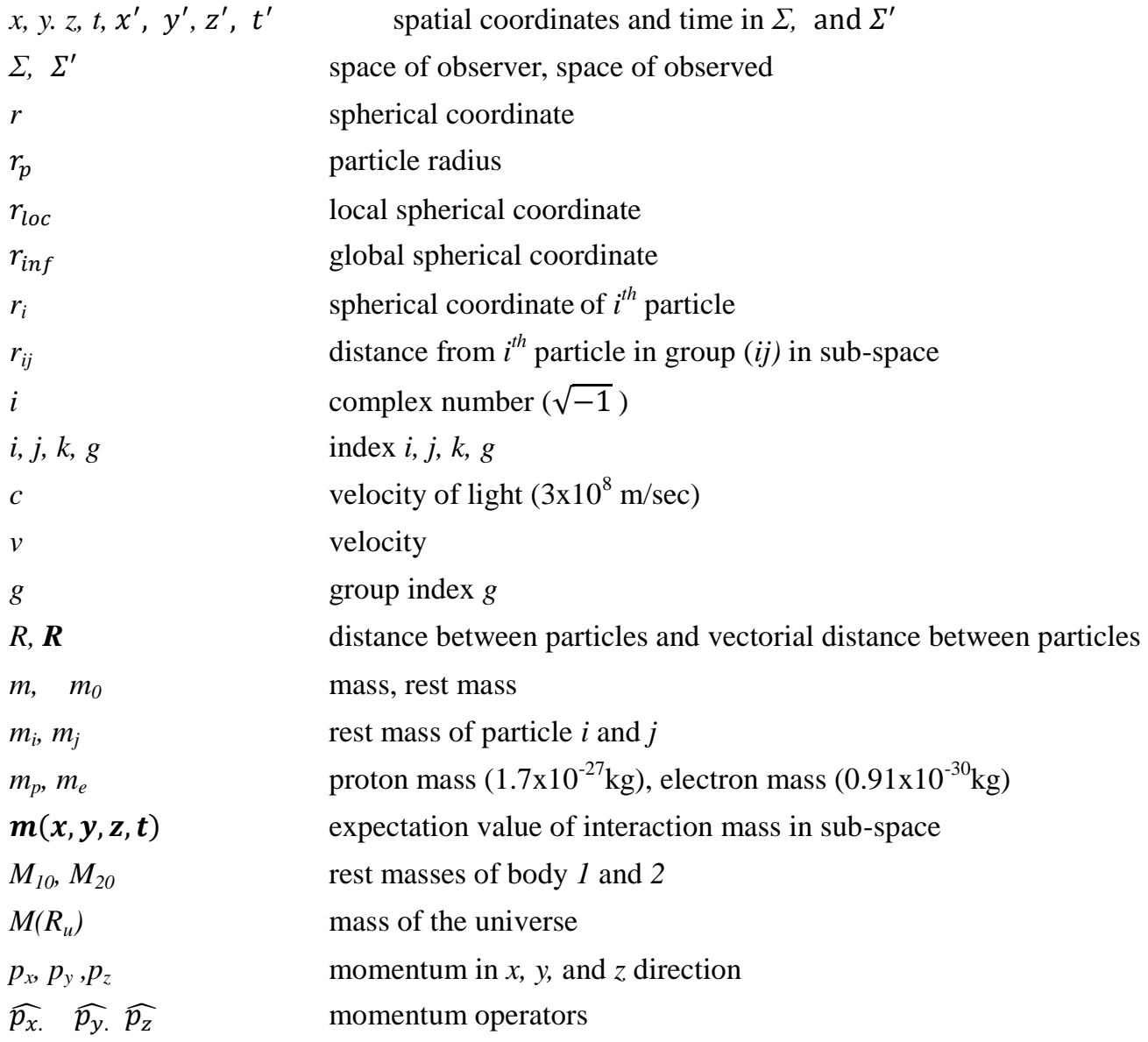




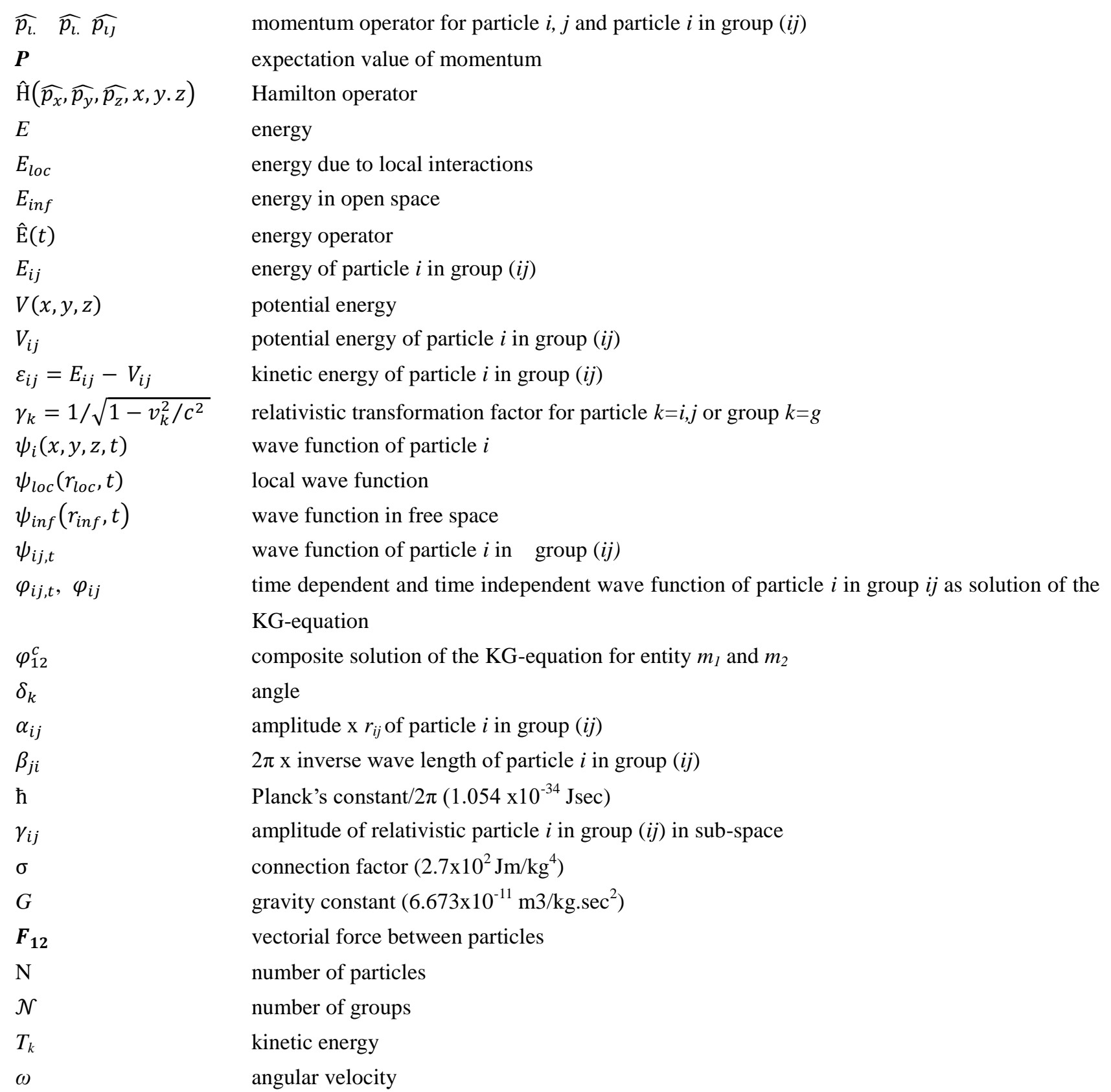

\section{Copyrights}

Copyright for this article is retained by the author(s), with first publication rights granted to the journal.

This is an open-access article distributed under the terms and conditions of the Creative Commons Attribution license (http://creativecommons.org/licenses/by/4.0/). 